\title{
ERROR ESTIMATES IN THE FAST MULTIPOLE METHOD FOR SCATTERING PROBLEMS PART 2: TRUNCATION OF THE GEGENBAUER SERIES *
}

\author{
Quentin Carayol ${ }^{1}$ and Francis Collino ${ }^{2}$
}

\begin{abstract}
We perform a complete study of the truncation error of the Gegenbauer series. This series yields an expansion of the Green kernel of the Helmholtz equation, $\frac{\mathrm{e}^{i|\vec{u}-\vec{v}|}}{4 \pi i \mid \vec{u}-\vec{v}}$, which is the core of the Fast Multipole Method for the integral equations. We consider the truncated series where the summation is performed over the indices $\ell \leq L$. We prove that if $v=|\vec{v}|$ is large enough, the truncated series gives rise to an error lower than $\epsilon$ as soon as $L$ satisfies $L+\frac{1}{2} \simeq v+C W^{\frac{2}{3}}\left(K(\alpha) \epsilon^{-\delta} v^{\gamma}\right) v^{\frac{1}{3}}$ where $W$ is the Lambert function, $K(\alpha)$ depends only on $\alpha=\frac{|\vec{u}|}{|\vec{v}|}$ and $C, \delta, \gamma$ are pure positive constants. Numerical experiments show that this asymptotic is optimal. Those results are useful to provide sharp estimates of the error in the fast multipole method for scattering computation.
\end{abstract}

Mathematics Subject Classification. 33C10, 33C55, 41A80.

Received: October 26, 2004.

\section{INTRODUCTION}

\subsection{Motivation}

This paper is the second one of a series of three, addressing the analysis of the error in the Fast Multipole Method (FMM) for scattering problems. Since the pioneer work of Rokhlin, the FMM has been proved to be a very effective tool for solving 3-D acoustic or electromagnetic scattering problems. This method rests on an approximation of the fundamental solution to the Helmholtz equation with a series of multipoles (known as Gegenbauer's identity). Let $\vec{u}, \vec{v}$ be two vectors of $\mathbb{R}^{3}$ with respective modulae $u$ and $v$, and respective directions $\hat{u}$ and $\hat{v}: \vec{u}=u \hat{u}, \vec{v}=v \hat{v}$. With these notations Gegenbauer's identity reads

$$
\frac{\mathrm{e}^{i|\vec{u}-\vec{v}|}}{i|\vec{u}-\vec{v}|} \simeq \sum_{\ell=0}^{L}(2 \ell+1) j_{\ell}(v) h_{\ell}^{(1)}(u) P_{\ell}(\hat{u} \cdot \hat{v}), \quad v<u
$$

\footnotetext{
Keywords and phrases. Gegenbauer, fast multipole method, truncation error.

* Supported by Dassault Aviation.

1 Dassault Aviation, 78 quai Marcel Dassault, Cedex 300, 92552 Saint-Cloud Cedex, France. quentin. carayol@dassault-aviation.fr

2 CERFACS, 42 avenue G. Coriolis, 31057 Toulouse, France. Collino@cerfacs.fr
} 
or, equivalently,

$$
\frac{\mathrm{e}^{i|\vec{u}-\vec{v}|}}{i|\vec{u}-\vec{v}|} \simeq \frac{1}{4 \pi} f_{S^{2}}\left(\sum_{\ell=0}^{L}(2 \ell+1) i^{\ell} h_{\ell}^{(1)}(u) P_{\ell}(\hat{u} \cdot \hat{v})\right) \mathrm{e}^{i \vec{v} \cdot \hat{s}} \mathrm{~d} \sigma(\hat{s}), \quad v<u .
$$

Here, $j_{\ell}$ and $h_{\ell}^{(1)}$ are the spherical Bessel and Hankel functions, $P_{\ell}$ the Legendre polynomials, and $f_{S^{2}} \mathrm{~d} \sigma(\hat{s})$ stands for some quadrature rule on the sphere $S^{2}$, see Darve [12] or Chew et al. [8] for more details, and [13] for another multipole formula. The error in this approximation is controlled by both the number of multipoles, $L$, and the choice of the quadrature rule. Greengard and Rokhlin were the first authors to provide empirical laws for the truncation integer $L$ that achieves a given precision, at least when $v$ is not too large. Those formulas have been fixed and improved by Chew and Song [17], (see also Chew [8]), but with no precise analytical error estimates. On the other hand, Rahola [21] then Darve [11], gave precise results, i.e. bounds of the truncation error as function of $L$ but their results lead to overestimate the value of $L$. It is precisely the goal of our study to provide true estimate errors that give the optimal values of $L$. The calculations that we were led to do are rather long and technical (see the Ph.D. [5] for an overview). This is the reason why we chose to divide this study into three different parts. In the first one [6], we studied the truncation error in the series of formula (1) when $u$ is large, which amounts to analyze the Jacobi-anger series. This first paper contains some techniques and results which will be useful in the present article, devoted to the truncation error for finite $u$. Eventually, the error due to the quadrature law in (2) will be the topic of a third article.

After this general presentation, we turn now to the topic of the present paper: the analysis of the truncation of the Gegenbauer series.

\subsection{The Gegenbauer series}

We will analyze the Gegenbauer's identity in the following form

$$
\frac{\mathrm{e}^{i|\vec{u}+\vec{v}|}}{i|\vec{u}+\vec{v}|}=\sum_{\ell=0}^{\infty}(-1)^{\ell}(2 \ell+1) h_{\ell}^{(1)}(u) j_{\ell}(v) P_{\ell}(\hat{u} \cdot \hat{v}), \quad \forall \vec{u}, \vec{v}, u>v .
$$

In this identity, several special functions take place

- $j_{\ell}, h_{\ell}^{(1)}=j_{\ell}+i y_{\ell}$, the spherical Bessel and Hankel functions of order $\ell$. They are linked to ordinary Bessel functions of first and second kind by

$$
j_{\ell}(t)=\sqrt{\frac{\pi}{2 t}} J_{\ell+\frac{1}{2}}(t), \quad y_{\ell}(t)=\sqrt{\frac{\pi}{2 t}} Y_{\ell+\frac{1}{2}}(t) ;
$$

- $P_{\ell}(x)$, the Legendre polynomial of order $\ell$. Two important properties of these polynomials are $[18,19]$

$$
\begin{gathered}
\left|P_{\ell}(x)\right| \leq 1, \quad \text { for all } x \in[-1,1], \quad P_{\ell}(1)=1, \quad P_{\ell}(-1)=(-1)^{\ell} \\
\left.P_{\ell}(x) \leq \sqrt{\frac{2}{\pi\left(\ell+\frac{1}{2}\right)}} \frac{1}{\left(1-x^{2}\right)^{\frac{1}{4}}}, \text { for all } x \in\right]-1,1[.
\end{gathered}
$$

For a precise definition of these functions and of their properties, the reader can refer to $[1,10,15,20,22]$ for instance.

A large number of authors have already dealt with the truncation error of that series. Among them, Greengard [9] gave an empirical formula for finding the truncation integer $L$ yielding an error $\epsilon$ :

$$
L=v+C(\epsilon) \ln (v+\pi) .
$$


Rahola [21] showed that the series was bounded by a geometrical series, Darve [11] analyzed more precisely the absolute error, and Koc et al. [17] gave some elements of analysis, and mentioned the formula

$$
L=v+C(\epsilon) v^{\frac{1}{3}}
$$

with some justifications but no rigorous proof. The aim of this work is to perform a systematic analysis of the truncation error and especially to provide an asymptotic formula of the truncation integer when $v$ goes to infinity.

\subsection{Qualitative description of the error}

We choose to examine separately the real and imaginary parts of the series, that is to say

$$
\left\{\begin{array}{l}
e_{c}(\vec{u}, \vec{v}, L)=|\cos | \vec{u}+\vec{v}|+| \vec{u}+\vec{v}\left|\sum_{\ell=0}^{L}(-1)^{\ell}(2 \ell+1) y_{\ell}(u) j_{\ell}(v) P_{\ell}(\hat{u} \cdot \hat{v})\right| \\
e_{s}(\vec{u}, \vec{v}, L)=|\sin | \vec{u}+\vec{v}|-| \vec{u}+\vec{v}\left|\sum_{\ell=0}^{L}(-1)^{\ell}(2 \ell+1) j_{\ell}(u) j_{\ell}(v) P_{\ell}(\hat{u} \cdot \hat{v})\right| .
\end{array}\right.
$$

This choice is due to a difference of behaviour between these series: it is not necessary to have $u>v$ for the convergence of the sine part, and, in a practical point of view, it is always much more difficult to have the cosine part converged. That is why we will focus on $e_{c}$ in the sequel. Note that (9) can be viewed as relative errors since $1 /|u+v|$ is the modulus of the approximated quantity (i.e. the Green function).

First and foremost, we get rid of the spherical functions and we rewrite Gegenbauer's identity in the form:

$$
e_{c}(\vec{u}, \vec{v}, L)=\frac{\pi}{2} \frac{|\vec{u}+\vec{v}|}{\sqrt{u v}}\left|\sum_{\ell=L+1}^{\infty}(-1)^{\ell}(2 \ell+1) J_{\ell+\frac{1}{2}}(v) Y_{\ell+\frac{1}{2}}(u) P_{\ell}(\hat{u} \cdot \hat{v})\right| .
$$

To get a uniform bound over all directions $\hat{u}$ and $\hat{v}$, we may use (5) and obtain

$$
e_{c}(\vec{u}, \vec{v}, L) \leq e_{c}^{a b s}(u, v, L) \text { with } \quad e_{c}^{a b s}(u, v, L)=\frac{\pi}{2} \frac{\alpha+1}{\sqrt{\alpha}}\left(\sum_{\ell=L+1}^{\infty}(2 \ell+1)\left|J_{\ell+\frac{1}{2}}(v)\right|\left|Y_{\ell+\frac{1}{2}}(u)\right|\right)
$$

where $\alpha$ is defined by

$$
\alpha=\frac{u}{v} \geq 1
$$

This series converges uniformly on every compact set of $(u, v), 0 \leq v<u$, since (cf. [10], p. 28)

$$
Y_{\ell+\frac{1}{2}}(u)=-\left(\frac{2}{u}\right)^{\ell+\frac{1}{2}} \frac{\Gamma\left(\ell+\frac{1}{2}\right)}{\pi}\left(1+o\left(\frac{1}{\ell+\frac{1}{2}}\right)\right), \quad J_{\ell+\frac{1}{2}}(v)=\left(\frac{v}{2}\right)^{\ell+\frac{1}{2}} \frac{1}{\Gamma\left(\ell+\frac{3}{2}\right)}\left(1+o\left(\frac{1}{\ell+\frac{1}{2}}\right)\right)
$$

whence we deduce the existence of constants $C_{j}(v)$ and $C_{y}(u)$ such that

$$
\pi\left|Y_{\ell+\frac{1}{2}}(u)\right| \leq C_{y}(u)\left(\frac{2}{u}\right)^{\ell+\frac{1}{2}} \Gamma\left(\ell+\frac{1}{2}\right) \quad \text { and } \quad\left|J_{\ell+\frac{1}{2}}(v)\right| \leq C_{j}(v)\left(\frac{v}{2}\right)^{\ell+\frac{1}{2}} \frac{1}{\Gamma\left(\ell+\frac{1}{2}\right)\left(\ell+\frac{1}{2}\right)}
$$

which leads to

$$
\frac{\pi}{2}(2 \ell+1)\left|Y_{\ell+\frac{1}{2}}(u)\right|\left|J_{\ell+\frac{1}{2}}(v)\right| \leq C_{j}(v) C_{y}(u)\left(\frac{v}{u}\right)^{\ell+\frac{1}{2}}
$$



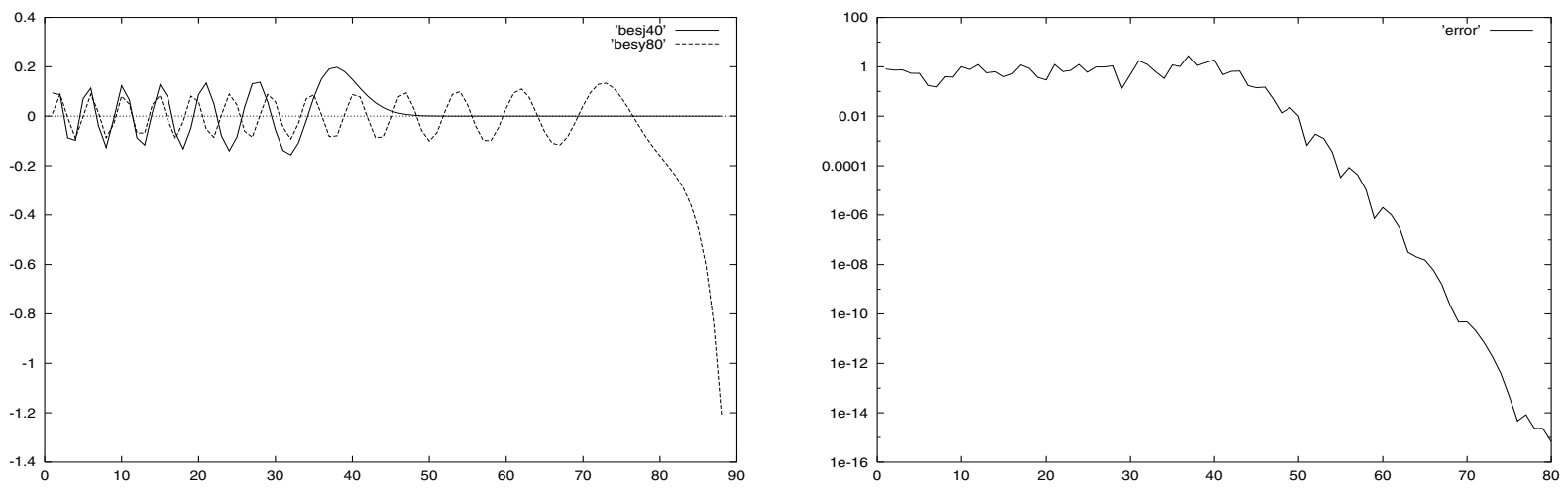

FigurE 1. Left: Bessel functions $J_{\ell+\frac{1}{2}}(40)$ and $Y_{\ell+\frac{1}{2}}(80)$ with respect to $\ell$. Right: Error $e_{c}(40 \hat{x}, 80 \hat{x}, L)$ with respect to $L$.

bounding the general term of the series by a geometric sequence of ratio $\frac{1}{\alpha}$. It leads to the upper bound

$$
e_{c}^{a b s}(u, v, L) \leq C_{j}(v) C_{y}(u) \frac{1+\alpha}{\sqrt{\alpha}} \sum_{\ell \geq L+1} \frac{1}{\alpha}^{\ell+\frac{1}{2}}=C_{j}(v) C_{y}(u) \frac{1+\alpha}{1-\alpha}\left(\frac{1}{\alpha}\right)^{L+1} .
$$

Of course this upper bound does not give satisfactory results when $u$ and $v$ go to infinity. Indeed, if $C_{j}(v)$ is uniformly bounded over $\mathbb{R}^{+}$(in fact $C_{j}(v)$ is smaller than $1[22]$ ), it is surely not the case for $C_{y}(u$ ), as mentioned by Darve in [11]. In order to obtain some estimates for large values of $u$ and $v$, we need to use sharper arguments concerning Bessel functions. Following Darve [11], we first give an example which is particular, but quite representative of the general situation. The variations of the sequences $J_{\ell+\frac{1}{2}}(40)$ and $Y_{\ell+\frac{1}{2}}(80)$ are plotted in Figure 1, left, whereas Figure 1, right, shows the relative error $e_{c}(40 \hat{x}, 80 \hat{x}, L)$ as a function of $L$. The sequence $J_{\ell+\frac{1}{2}}(40)$ first oscillates, then from an integer $\ell_{40} \simeq 45$, it abruptly goes to 0 . The sequence $Y_{\ell+\frac{1}{2}}(80)$ looks similar for small values of $\ell$ : it smoothly oscillates, but as soon as $\ell$ exceeds $\ell_{80} \simeq 78$, it becomes negative and goes violently to $-\infty$. We also see that the error starts decreasing only when $L$ exceeds $\ell_{40}$, that is to say when $J_{\ell+\frac{1}{2}}(40)$ stops oscillating. From this integer, the decrease is seemingly faster than exponential. These curves indicate that an appropriate zone of error study is for $L$ slightly greater than $v$. That is why we will always assume $L+\frac{1}{2} \geq v$, which implies (see Prop. 2.3 hereunder)

$$
J_{\ell}(v) \geq 0, \forall \ell \geq L .
$$

Moreover, these curves show that it is necessary to draw two distinct analysis, for $\ell$ such that $Y_{\ell+\frac{1}{2}}(u)$ oscillates and for the explosion zone. Now we know that, if $y_{\nu, 1}$ is the first zero of the function $t \rightarrow Y_{\nu}(t)$, then $\left\{y_{\nu, 1}\right\}_{\nu}$ is a decreasing sequence of $\nu$ and we have [1]

$$
y_{\nu, 1} \geq \nu+1, \text { when } \nu \geq \frac{1}{2} \quad \text { and } \quad y_{\nu, 1} \sim \nu+0.9368 \ldots \nu^{\frac{1}{3}}+\ldots \nu^{-\frac{2}{3}}+\ldots
$$

It is even true that $y_{\nu, 1} \geq \nu+2$ as soon as $\nu$ exceeds some threshold, which is located, according to the tables, between 7 and 8 . Before that first zero, $Y_{\nu}(t) \leq 0, t \leq y_{\nu, 1}$ and we thus have

$$
Y_{\ell+\frac{1}{2}}(u)<0 \text { when } u \leq \ell+\frac{3}{2} \quad \text { while } \quad Y_{\ell-\frac{1}{2}}(u)<0 \text { when } u \leq \ell+\frac{3}{2} \text { and } 8 \leq \ell \text {, }
$$


which characterizes the explosion zone. Let us choose an integer $N$ such that $u \leq N+\frac{5}{2}$, then we can get rid of the absolute values and write

$$
e_{c}^{a b s}(u, v, N)=-\frac{\pi}{2} \frac{\alpha+1}{\sqrt{\alpha}}\left(\sum_{\ell=N+1}^{\infty}(2 \ell+1) J_{\ell+\frac{1}{2}}(v) Y_{\ell+\frac{1}{2}}(u)\right) .
$$

These results lead us to define a pivot value $N_{u}$ depending on $u$

$$
N_{u}=\left[u-\frac{1}{2}\right]=\left[\alpha v-\frac{1}{2}\right]
$$

and to differentiate our analyze according to whether $L$ is chosen lower or greater than $N_{u}$. This pivot value will be relevant only if $u$ is greater than $\frac{1}{2}$, which we will always assume from now on. We will also impose the constraint

which automatically holds when

$$
v \leq N_{u}+\frac{1}{2}
$$

$$
u=\alpha v \geq \frac{\alpha}{\alpha-1} \quad(\geq 1) .
$$

Let $\epsilon$ be some error criterion to reach. If $u$ is "large enough" we will begin by estimating $e_{c}^{a b s}\left(u, v, N_{u}\right)$. Then two different cases appear:

- $e_{c}^{a b s}\left(u, v, N_{u}\right) \geq \epsilon$ : this case corresponds to $\frac{1}{\alpha-1}$ large or $\epsilon$ very small. A numerical difficulty is related to that case. Indeed, taking $L$ greater than $N_{u}$ requires to use some $Y_{\ell+\frac{1}{2}}(u)$ where the argument of the Bessel function exceeds its index, so that we work in what we called the explosion zone. Then some problems of computer accuracy occur in the representation of numbers, since we have to deal with large numbers the sum of which must be small. This phenomenon is commonly referred to as the 'numerical breakdown'. It was mentioned in many articles about the Fast Multipole Method [11,14];

- $e_{c}^{a b s}\left(u, v, N_{u}\right) \leq \epsilon$ : this is the "usual" case, when $\frac{1}{\alpha-1}$ and $\frac{1}{\epsilon}$ are not too large compared with $u$. In this situation, we can take $L$ smaller than $N_{u}$. Again we will distinguish a case when $u$ is "moderately large" and a case when it is "very large".

We will study the last case more particularly to find asymptotic results. Indeed, when $u$ (or $v$ ) is large, it is known (and we will show) that the convergence of the series is fast enough to occur before the breakdown phenomenon (i.e. for a $L$ lower than $N_{u}$ ).

\subsection{Description of our results}

We first analyze the series $e_{c}^{a b s}(u, v, L)$ defined in (10), which constitutes a uniform upper bound of $e_{c}(\vec{u}, \vec{v}, L)$, then we study the series $e_{c}(\vec{u}, \vec{v}, L)$.

Let $\beta$ be the angle defined by the two vectors $\vec{u}$ and $\vec{v}$, i.e. $\cos \beta=\hat{u} \cdot \hat{v}$. The main results of the paper can be described in the following way. We derive laws in the form $L_{a b s}(v, \alpha, \epsilon)$ and $L_{\beta}(v, \alpha, \epsilon)$ such that

$$
\begin{aligned}
& \frac{\pi}{2} \frac{\alpha+1}{\sqrt{\alpha}} \sum_{\ell=L_{\mathrm{abs}}(v, \alpha, \epsilon)+1}^{\infty}(2 \ell+1)\left|J_{\ell+\frac{1}{2}}(v)\right|\left|Y_{\ell+\frac{1}{2}}(u)\right| \leq \epsilon, \\
& \frac{\pi}{2} \frac{|\vec{u}+\vec{v}|}{\sqrt{u v}}\left|\sum_{\ell=L_{\beta}(v, \alpha, \epsilon)+1}^{\infty}(2 \ell+1) J_{\ell+\frac{1}{2}}(v) Y_{\ell+\frac{1}{2}}(u) P_{\ell}(\cos \beta)\right| \leq \epsilon
\end{aligned}
$$

with the asymptotics (subscript $*$ stands for abs or $\beta$ )

$$
L_{*}(v, \alpha, \epsilon) \simeq v+C_{*} W^{\frac{2}{3}}\left(K_{*}(\alpha) \frac{v^{\gamma_{*}}}{\epsilon^{\delta_{*}}}\right) v^{\frac{1}{3}}-\frac{1}{2} \text { plus terms vanishing with } v \text { when } v \text { is large, }
$$


TABLE 1. Values of the constants involved in the asymptotic formula (16) for different configurations.

\begin{tabular}{|c||c|c|c|c|c|}
\hline & $C_{*}$ & $K_{*}(\alpha)$ & $\gamma_{*}$ & $\delta_{*}$ & $c f$. formula \\
\hline \hline$L_{a b s}(v, \alpha, \epsilon)$ & $\frac{1}{2}\left(\frac{3}{2}\right)^{\frac{2}{3}}$ & $\frac{2(1+\alpha)^{2}}{3 \alpha \sqrt{\alpha^{2}-1}}$ & 1 & 2 & $(57)$ \\
$L_{\beta}(v, \alpha, \epsilon), \cos \beta=1$ & $\left(\frac{1}{2}\right)^{\frac{5}{3}}$ & $\frac{1}{4}\left(\frac{1+\alpha}{1-\alpha}\right)^{\frac{3}{2}}$ & 1 & 6 & $(74)$ \\
$L_{\beta}(v, \alpha, \epsilon), \cos \beta=-1$ & $\left(\frac{1}{2}\right)^{\frac{5}{3}}$ & $\frac{1}{4}\left(\frac{1-\alpha}{1+\alpha}\right)^{\frac{3}{2}}$ & 1 & 6 & $(70)$ \\
$L_{\beta}(v, \alpha, \epsilon), \cos \beta=-\frac{1}{\alpha}$ & $\frac{1}{2}\left(\frac{3}{2}\right)^{\frac{2}{3}}$ & $\frac{4}{3 \pi}$ & 0 & 2 & Section 5.3 \\
\hline
\end{tabular}

the various constants being specific to the considered case: their precise values are given in Table 1. Here, function $W$ is the Lambert function

$$
W(t) \mathrm{e}^{W(t)}=t, \quad t>0
$$

a function with the sub-logarithmic behaviour $W(t)=\log (t / \log t)+o(1)$ when $t$ is large.

These laws appear to be asymptotically optimal. Note that no asymptotic formula is given for the cases $\cos \beta \neq-1,-\frac{1}{\alpha}$ or 1 . Our conjecture is that the relative error in those cases goes to 0 when $v$ goes to $\infty$, as soon as $L$ is greater than $v$, (see Sect. 5.3 for some arguments in this direction).

Furthermore, we prove that, if $L_{\text {unif }}$ is the truncation integer yielding a uniform bound of $\epsilon$ over all values of $\beta$, then $L_{\text {unif }}$ follows the same asymptotic expansion as $L_{\beta=0}(v, \alpha, \epsilon)$, i.e.

$$
L_{\text {unif }}(v, \alpha, \epsilon)+\frac{1}{2} \simeq v+\left(\frac{1}{2}\right)^{\frac{5}{3}} W^{\frac{2}{3}}\left(\left(\frac{1+\alpha}{1-\alpha}\right)^{\frac{3}{2}} \frac{v}{4 \epsilon^{6}}\right) v^{\frac{1}{3}}
$$

This is probably the most important result of the article, since it gives an accurate and uniform truncation rule for the true Gegenbauer series. Actually, we prove in a more general way that $L_{a b s}\left(v_{\max }, \alpha_{0}, \epsilon\right)$ and $L_{\text {unif }}\left(v_{\max }, \alpha_{0}, \epsilon\right)$ yield an error uniformly lower than $\epsilon$ for all $v<v_{\max }$ and $u>\alpha_{0} v_{\max }$.

All those results are important since, they give the key to understand some phenomena which were mysterious so far in the application of the Fast Multipole Method. On the one hand, they show how $L$ should be chosen (the answer being: $\left.L_{\text {unif }}\left(v_{\max }, \alpha_{0}, \epsilon\right)\right)$ and why previously used empirical formulas were almost right. On the other hand, Section 5 epitomizes the fact that just a few configurations of vectors $\hat{\mathbf{u}}$ and $\hat{\mathbf{v}}$, that is to say a few configurations of points in boxes of an oct-tree, oblige us to use such a constraining formula. That is why, even if people have always chosen looser formulas so far, numerical results in the FMM never showed an increasing error with the size of the boxes: the number of non particular points was always much too large with respect to particular points.

Remark 1.1. In the case of a multipole method using a cube-splitting oct-tree, we can actually deduce some interesting points from this study. For large sizes of cubes, the penalizing case is that of colinear vectors, in the direction of a diagonal of the cubes, with a ratio of two between their respective norms. It corresponds to points right in the corner of cubes, which is a rather rare event indeed.

Remark 1.2. The notion of relative error seems to us more adequate than the absolute error. However, when the absolute error is considered rather than the relative error as in [11], it can be proved that $L=[v]$ is sufficient to make the truncation error go to 0 . This result improves the result obtained by E. Darve, i.e. $L=C v+C^{\prime} \log (v)+C^{\prime \prime} \log \left(\epsilon^{-1}\right)$ with $C>1$.

All these results concern the asymptotic case, i.e. when $v$ is large enough. For finite $v$, we would like to emphasize one of our result, which relates to the case when $e_{c}^{a b s}\left(u, v, N_{u}\right) \geq \epsilon$ (or $L>N_{u}$ ). We prove the 
following estimate: if $L>N_{u}$ and $L>v$,

$$
e_{c}^{a b s}(u, v, L) \leq \frac{\alpha+1}{\alpha-1} \sqrt{\alpha} C_{\max } \frac{\nu}{\sqrt{\nu^{2}-u^{2}}} \mathrm{e}^{-\nu\left(F\left(\frac{v}{\nu}\right)-F\left(\frac{u}{\nu}\right)\right)}, \quad \nu=L+\frac{3}{2}, \quad F \text { given in (24) }
$$

where $C_{\max } \simeq 1.04$ and $F(x) \geq \frac{1}{3}\left(1-x^{2}\right)^{\frac{3}{2}}$. This results seems to be new.

\subsection{Outline}

Our whole article relies on several technical ingredients. First, we use some accurate estimates of all the involved special functions (Bessel and Hankel functions as well as Legendre polynomials). We develop all these results in the first part of Section 2. Another major ingredient is the derivation of a simple and explicit formula for the remainder of the Gegenbauer's series in some particular cases. This is the subject of the second part of Section 2. With these preliminary results, we are able to give some accurate estimates of the truncation error for the bounding series $e_{c}^{a b s}$. These various bounds, described in Section 3, depends on the location of the truncation integer with regard to the pivot value $N_{u}$. In Section 4 , we estimate the truncation integer $L_{a b s}(v, \alpha, \epsilon)$ and we establish its asymptotic expansion (Thm. 4.1). In Section 5, we study the behaviour of the truncation error of the initial Gegenbauer series with respect to the directions of $\hat{\mathbf{u}}$ and $\hat{\mathbf{v}}$. We show that four configurations yield different kinds of results, and we derive asymptotic rules for $L_{\beta}$ in the three most demanding cases (see the table in previous section). Finally, we give in Section 6 a uniform bound over all directions $\hat{\mathbf{u}}$ and $\hat{\mathbf{v}}$ yielding the final asymptotic expansion of $L_{\text {unif }}$.

\section{TECHNiCAL TOOLS}

This section is devoted to the presentation of the basic ingredients of our proofs. Accurate estimates and asymptotic formulas for Bessel functions are given. A useful explicit form for the Gegenbauer series is also derived.

\subsection{Estimates related to Bessel functions}

\subsubsection{Estimates for $Y_{\nu}(u)$ and $H_{\nu}^{(1)}(u)$}

We begin with the Bessel functions of second species. One of the important properties that we will use is

$$
\left|Y_{\nu}(t)\right| \leq\left|J_{\nu}(t)+i Y_{\nu}(t)\right|=\left|H_{\nu}^{(1)}(t)\right|
$$

thus estimates on $\left|H_{\nu}^{(1)}(t)\right|$ automatically give us estimates on $\left|Y_{\nu}(t)\right|$.

Proposition 2.1. The function $(u, \nu) \rightarrow \sqrt{\nu}\left|H_{\nu}^{(1)}(u)\right|$ is an increasing function in $\nu$ and a decreasing function in $u$ for $u>0, \nu>\frac{1}{2}$. Moreover, if $\nu \geq \frac{1}{2}$,

$$
\sqrt{\frac{2}{\pi u}} \leq\left|H_{\nu}^{(1)}(u)\right| \leq \sqrt{\frac{2}{\nu \pi}} \frac{1}{\left(\frac{u^{2}}{\nu^{2}}-1\right)^{\frac{1}{4}}}, \quad \text { if } u>\nu .
$$

Besides, there is a constant $C_{0}$ such that

$$
\left|H_{u}^{(1)}(u)\right| \leq \frac{C_{0}}{u^{\frac{1}{3}}}, \text { for } u \geq \frac{1}{2} .
$$

Proof. Inequality (19) can be found in [22]. It relies on Nicholson's formula ([22], p. 444)

$$
\left|H_{\nu}^{(1)}(u)\right|^{2}=\frac{8}{\pi^{2}} \int_{0}^{\infty} K_{0}(2 u \sinh t) \cosh (2 \nu t) \mathrm{d} t,
$$




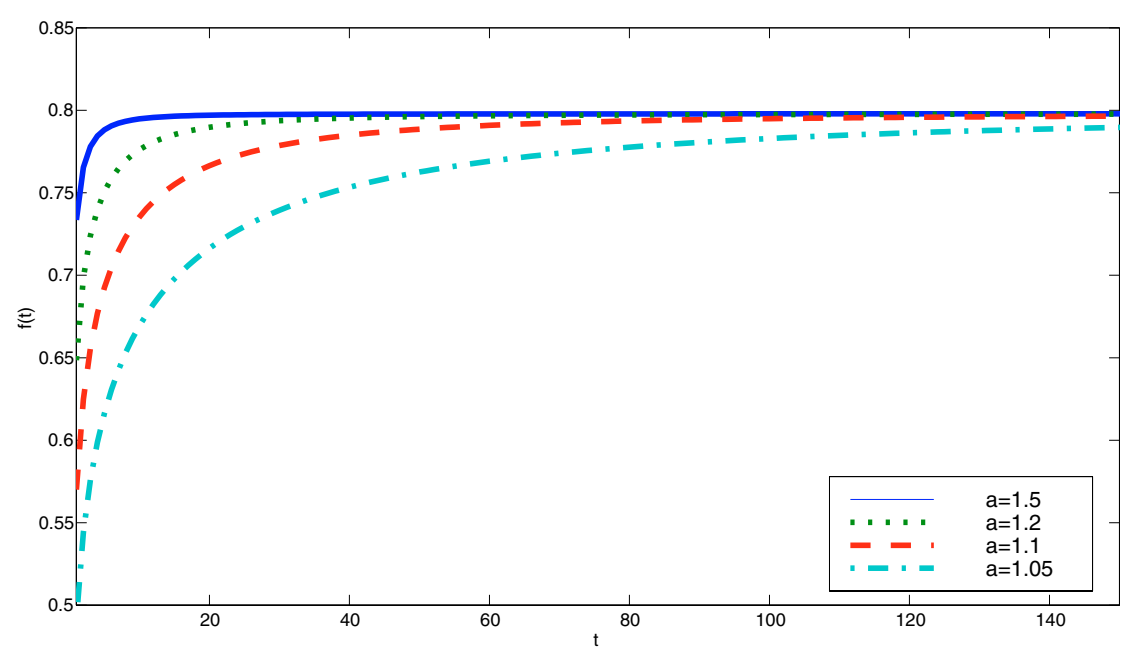

Figure 2. Function $f_{a}(t)=\left(a^{2}-1\right)^{\frac{1}{4}} \sqrt{t}\left|H_{t}^{(1)}(a t)\right|$.

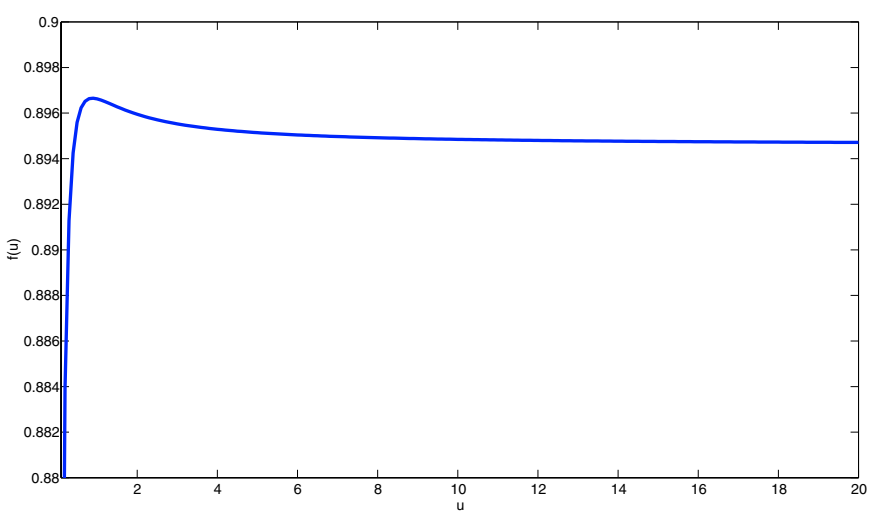

FigurE 3. Graph of function $f(u)=u^{\frac{1}{3}}\left|H_{u}^{(1)}(u)\right|$.

where $K_{0}(\xi)$ is the Kelvin's function

$$
K_{0}(\xi)=\int_{0}^{\infty} \mathrm{e}^{-\xi \cosh x} \mathrm{~d} x
$$

Figure 2 shows that the upper bound is a fairly accurate estimate of $\left|H_{\nu}^{(1)}(u)\right|$. Lastly, (20) comes from the behaviour ([22], p. 232)

$$
\lim _{\nu \rightarrow+\infty} \nu^{\frac{1}{3}} J_{\nu}(\nu)=\frac{\Gamma\left(\frac{1}{3}\right)}{2^{\frac{2}{3}} 3^{\frac{1}{6}} \pi}, \quad \lim _{\nu \rightarrow+\infty} \nu^{\frac{1}{3}} Y_{\nu}(\nu)=-\frac{3^{\frac{1}{6}} \Gamma\left(\frac{1}{3}\right)}{2^{\frac{2}{3}} \pi}
$$

By the way, a numerical computation (see Fig. 3) indicates that we have $C_{0} \leq 0.89666<1$. 
Proposition 2.2. Let $C>0, \delta \in] \frac{1}{3}, \frac{1}{2}\left[\right.$, and $\alpha_{0}>1$, then there exists some $V_{\delta, C, \alpha_{0}}$ and $C_{\alpha_{0}}$ such that for all $v$ larger than $V_{\delta, C, \alpha_{0}}$, all $\left.\nu \in\right] v-1, v\left[\right.$, all $u=\alpha v \geq \alpha_{0} v$ and all integer $p \leq C v^{\delta}$, we have

$$
\begin{gathered}
\left|\sqrt{\frac{\pi u}{2}} H_{\nu+p}^{(1)}(u)\right| \leq \frac{\sqrt{\alpha}}{\left(\alpha^{2}-1\right)^{\frac{1}{4}}}\left(1+\frac{C_{\alpha_{0}} C^{2}}{v^{1-2 \delta}}\right) \\
\left|\sqrt{\frac{\pi u}{2}}\left(H_{\nu+p+1}^{(1)}(u)+H_{\nu+p}^{(1)}(u)\right)\right| \leq \sqrt{2}\left(\frac{\alpha+1}{\alpha-1}\right)^{\frac{1}{4}}\left(1+\frac{C_{\alpha_{0}} C^{2}}{v^{1-2 \delta}}\right), \\
\left|\sqrt{\frac{\pi u}{2}}\left(H_{\nu+p+1}^{(1)}(u)-H_{\nu+p}^{(1)}(u)\right)\right| \leq \sqrt{2}\left(\frac{\alpha-1}{\alpha+1}\right)^{\frac{1}{4}}\left(1+\frac{C_{\alpha_{0}} C^{2}}{v^{1-2 \delta}}\right) .
\end{gathered}
$$

Proof. The proof relies on Theorem A.1 in Appendix A that allows us to replace $H_{\nu+q}^{(1)}(u)$ by $H_{\nu}^{(1)}(u) \mathrm{e}^{-i q \gamma}$ with $\cos \gamma=\frac{1}{\alpha}$ for $q<C v^{\delta}$ up to an error (uniform in $\alpha>\alpha_{0}$ ) negligible when $v$ tends to infinity. Since $\left|H_{\nu}^{(1)}(u)\right|$ increases with $\nu<v,(21)$ is a direct consequence of the bound (19) for $\left|H_{v}^{(1)}(u)\right|$. The two other inequalities are derived in the same way, with the additional term $\left|2 \cos \frac{\gamma}{2}\right|=\sqrt{2+\frac{2}{\alpha}}$ for the sum of two consecutive Hankel functions and the term $\left|2 \sin \frac{\gamma}{2}\right|=\sqrt{2-\frac{2}{\alpha}}$ for the difference.

\subsubsection{Estimates for $J_{\nu}(u)$}

We give some results concerning the functions $J_{\nu}(v)$ for $v \leq \nu$. For $x$ in $\left.] 0,1\right]$, we define

$$
\left\{\begin{array}{l}
F(x)=\frac{1}{2} \log \left(\frac{1-\sqrt{1-x^{2}}}{1+\sqrt{1-x^{2}}}\right)-\sqrt{1-x^{2}} \quad \text { or, equivalently, } \\
F(x)=\int_{x}^{1} \sqrt{1-\xi^{2}} \frac{d \xi}{\xi}=\sum_{p=1}^{\infty} \frac{\left(1-x^{2}\right)^{\frac{2 p+1}{2}}}{2 p+1} \geq \frac{1}{3}\left(1-x^{2}\right)^{\frac{3}{2}}
\end{array}\right.
$$

Proposition 2.3. For $0<v<\nu$, the function $(v, \nu) \rightarrow J_{\nu}(v)$ is a positive function decreasing in $\nu$ and increasing in $v$. Moreover, if $u, v, \nu$ are such that $0<v \leq u \leq \nu$, then

$$
\begin{gathered}
0<J_{\nu}(v) \leq \frac{\mathrm{e}^{-\nu F\left(\frac{v}{\nu}\right)}}{\sqrt{2 \pi \nu}\left(1-\frac{v^{2}}{\nu^{2}}\right)^{\frac{1}{4}}}, \\
\frac{J_{\nu}(v)}{J_{\nu}(u)} \leq \mathrm{e}^{-\nu\left(F\left(\frac{v}{\nu}\right)-F\left(\frac{u}{\nu}\right)\right)} \leq\left(\frac{v}{u}\right)^{\sqrt{\nu^{2}-u^{2}}} \\
\frac{v}{2} J_{\nu}(v) \leq(\nu+1) J_{\nu+1}(v) .
\end{gathered}
$$

Remark 2.4. Inequality (26) seems to be new.

Proof. Denote $y=\frac{u}{\nu}, x=\frac{v}{\nu}$, then $x<y<1$ by hypothesis. If $w$ stands for $u$ or $v, w \leq \nu$; we start from the formula, (see [22], p. 253)

$$
J_{\nu}(w)=\frac{1}{\pi} \int_{0}^{\pi} \mathrm{e}^{-\nu \tilde{F}\left(\theta, \frac{w}{\nu}\right)} \mathrm{d} \theta
$$

where

$$
\tilde{F}(\theta, z)=\log \left(\frac{\theta+\sqrt{\theta^{2}-z^{2} \sin ^{2} \theta}}{z \sin \theta}\right)-\frac{\cos \theta}{\sin \theta} \sqrt{\theta^{2}-z^{2} \sin ^{2} \theta}
$$


(note that $\tilde{F}(0, z)=F(z)$, with $F$ the function defined above). Since

$$
\tilde{F}(\theta, z) \geq 0, \quad \frac{\partial \tilde{F}}{\partial z}(\theta, z)=-\frac{\theta-z^{2} \sin \theta \cos \theta}{z \sqrt{\theta^{2}-z^{2} \sin ^{2} \theta}}=-\frac{g(\theta, z)}{z} \leq 0,
$$

$w \rightarrow J_{\nu}(w)$ is a decreasing function in $\nu$ and an increasing function in $w$, if $w \leq \nu$. Watson also proves that

$$
\tilde{F}(\theta, z) \geq F(z)+\frac{\theta^{2}}{2} \sqrt{1-x^{2}}
$$

from which he deduces (25). By the way, he shows that the extrema of function $g(\theta, z)$ over $[0, \pi]$ are $1=g(\pi, z)$, $\sqrt{1-z^{2}}=g(0, z)$ and, possibly, $\sqrt{1-\cos \left(2 \theta_{0}\right) z^{2}}$ for some $\theta_{0}$. Thus, we have

$$
\sqrt{1-z^{2}} \leq g(\theta, z) \leq \sqrt{1+z^{2}}
$$

Hence, since $\tilde{F}(\theta, z)$ is a decreasing function of $z$, we have

$$
\tilde{F}(\theta, x)-\tilde{F}(\theta, y)=-\int_{x}^{y} \frac{\partial \tilde{F}}{\partial z}(\theta, z) \mathrm{d} \theta=\int_{x}^{y} g(\theta, z) \frac{\mathrm{d} z}{z} \geq \int_{x}^{y} \sqrt{1-z^{2}} \frac{\mathrm{d} z}{z}=F(y)-F(x) \geq \sqrt{1-y^{2}} \log \frac{y}{x} .
$$

Considering (28) again, we obtain

$$
\begin{aligned}
J_{\nu}(v) & =\frac{1}{\pi} \int_{0}^{\pi} \mathrm{e}^{-\nu \tilde{F}(\theta, y)} \mathrm{e}^{-\nu(\tilde{F}(\theta, x)-\tilde{F}(\theta, y))} \mathrm{d} \theta \leq \mathrm{e}^{-\nu \inf _{\theta}(\tilde{F}(\theta, x)-\tilde{F}(\theta, y))} \frac{1}{\pi} \int_{0}^{\pi} \mathrm{e}^{-\nu \tilde{F}(\theta, y)} \mathrm{d} \theta \\
\text { and } J_{\nu}(v) & \leq \mathrm{e}^{-\nu(F(x)-F(y))} J_{\nu}(u) \leq \mathrm{e}^{-\nu \sqrt{1-y^{2}} \log \frac{y}{x}} J_{\nu}(u),
\end{aligned}
$$

that is exactly (26). Eventually, (27) is a known inequality, cf. [2] or [11], which can be inferred from $v\left(J_{\nu}(v)+\right.$ $\left.J_{\nu+2}(v)\right)=2(\nu+1) J_{\nu+1}(v)$ with $J_{\nu}(v), J_{\nu+1}(v), J_{\nu+2}(v)$ positive if $v \leq \nu$.

Proposition 2.5. Let $\nu, v_{\max }$ two positive numbers such that $v_{\max } \leq \nu$, then

$$
\begin{gathered}
\sup _{v \leq v_{\max }} \sqrt{\frac{\pi v}{2}} J_{\nu}(v) \leq \frac{\mathrm{e}^{-\frac{v_{\max }}{3} \Theta(x)}}{2 \Theta(x)^{\frac{1}{6}}}, \sup _{v \leq v_{\max }} \sqrt{\frac{\pi v}{2}}\left(J_{\nu}(v)-J_{\nu+1}(v)\right) \leq \frac{\mathrm{e}^{-\frac{v_{\max }}{3} \Theta(x)}}{2^{\frac{1}{4}} \sqrt{x}}, \text { with } x=\frac{v_{\max }}{\nu} \text { and } \\
\Theta(x)=\frac{\left(1-x^{2}\right)^{\frac{3}{2}}}{x} .
\end{gathered}
$$

Proof. The first estimate in (30) is a consequence of (25) with $F(x) / x \geq \frac{1}{3} \Theta(x)$ and $\sqrt{x} \leq x^{\frac{1}{6}}$ (note that $\left.x^{\frac{1}{6}}\left(1-x^{2}\right]^{\frac{1}{4}}=\Theta(x)^{\frac{1}{6}}\right)$. The second one is proved as follows; since (see [22], p. 55), $J_{\nu}^{\prime}(v)=\frac{\nu}{v} J_{\nu}(v)-J_{\nu+1}(v)$ and $J_{\nu+1}^{\prime}(v)=-\frac{\nu+1}{v} J_{\nu+1}(v)+J_{\nu}(v)$ we deduce that $\left(\sqrt{v}\left(J_{\nu}(v)-J_{\nu+1}(v)\right)\right)^{\prime}=\sqrt{v}\left(\frac{\nu+\frac{1}{2}}{v}-1\right)\left(J_{\nu+1}(v)+J_{\nu}(v)\right)$ is positive and the maximum of the function $\xi(v)=\sqrt{v}\left(J_{\nu}(v)-J_{\nu+1}(v)\right)$ is reached at $v=v_{\max }$. From $J_{\nu}(v) \leq \frac{\nu}{v} J_{\nu}(v)=\frac{1}{2}\left(J_{\nu-1}(v)+J_{\nu+1}(v)\right)$, we infer $\xi(v) \leq \frac{\sqrt{v}}{2}\left(J_{\nu-1}(v)-J_{\nu+1}(v)\right)=\sqrt{v} J_{\nu}^{\prime}(v)$. The result follows from, (see [22], p. 255), $x J_{\nu}^{\prime}(\nu x) \sqrt{2 \pi \nu} \leq \mathrm{e}^{-\nu F(x)}\left(1+x^{2}\right)^{\frac{3}{4}}$ and $\left(1+x^{2}\right)^{\frac{3}{4}} \leq 2^{\frac{3}{4}}$.

\subsubsection{Estimates for the remainder of the Jacobi Anger Series}

We recall here some results we obtained in [6] for the Jacobi-Anger series, or more precisely for a bounding series. 
Proposition 2.6. For all $v>0$ and $L>0$,

$$
\sqrt{\frac{\pi}{2 v}} \sum_{\ell=L+1}^{\infty}(2 \ell+1) J_{\ell+\frac{1}{2}}(v) \leq \sqrt{x} \frac{\mathrm{e}^{-v \frac{F(x)}{x}}}{\left(1-x^{2}\right)^{\frac{3}{4}}} \leq \frac{\mathrm{e}^{-\frac{v}{3} \Theta(x)}}{\sqrt{\Theta(x)}} \text {, with } x=\frac{v}{L+\frac{1}{2}}
$$

where the function denoted $\Theta(x)$ is defined in (31).

Let $\epsilon$ be a small positive real number; the equation $\frac{\mathrm{e}^{-\frac{v}{3} \Theta\left(x^{\epsilon}(v)\right)}}{\sqrt{\Theta\left(x^{\epsilon}(v)\right)}}=\epsilon$ is equivalent to

$$
\Theta\left(x^{\epsilon}(v)\right)=\frac{3}{2 v} W\left(\frac{2 v}{3 \epsilon^{2}}\right)
$$

where $W(x)$ is the Lambert function defined by

$$
W(x) \mathrm{e}^{W(x)}=x, \quad x>0 .
$$

For any $v$, the second inequality of (32) allows us to determine $\Theta\left(x^{\epsilon}(v)\right)$ by using (33), and then $x^{\epsilon}(v)$. When $v$ becomes large, $\Theta\left(x^{\epsilon}(v)\right)$ goes to $0, x^{\epsilon}(v)$ goes to 1 , and the following asymptotic development holds:

$$
x_{\epsilon}(v)=1-\frac{1}{2}\left(\frac{3}{2 v}\right)^{\frac{2}{3}} W^{\frac{1}{3}}\left(\frac{2 v}{3 \epsilon^{2}}\right)+\mathcal{O}\left(v^{-\frac{4}{3}} W^{\frac{4}{3}}\left(\frac{2 v}{3 \epsilon^{2}}\right)\right),
$$

which yields for $L$

$$
L_{\epsilon}(v)+\frac{1}{2}=v+\frac{1}{2}\left(\frac{3}{2}\right)^{\frac{2}{3}} v^{\frac{1}{3}} W^{\frac{2}{3}}\left(\frac{2 v}{3 \epsilon^{2}}\right)+\mathcal{O}\left(v^{-\frac{1}{3}} W^{\frac{4}{3}}\left(\frac{2 v}{3 \epsilon^{2}}\right)\right) .
$$

This formula gives an upper bound of the true $L$ for large values of $v$. However, according to the numerical results we presented in [6], it seems to be optimal.

\subsection{Explicit formula for the Gegenbauer series}

In this paragraph, we show that it is possible, in two special cases $(\vec{u}$ and $\vec{v}$ pointing in the same direction or in opposite directions) to transform the remainder of the Gegenbauer series into a simple and explicit form. This will provide us a tool for obtaining a sharp analysis of the error of truncature.

Proposition 2.7. Let $v>0, \alpha>1$ and $t \in[-1: 1]$ then for all $N \geq 0$ the following equality holds:

$$
\begin{aligned}
& (1-\alpha t) \sum_{\ell=N+1}^{\infty}(2 \ell+1) J_{\ell+\frac{1}{2}}(v) \mathcal{B}_{\ell+\frac{1}{2}}(\alpha v) P_{\ell}(t)=S_{N}(v, \alpha, t)+R_{N}(v, \alpha, t) \\
& \text { with } R_{N}(v, \alpha, t)=\alpha v\left(\mathcal{B}_{N+\frac{1}{2}}(\alpha v) J_{N+\frac{3}{2}}(v) P_{N+1}(t)-\mathcal{B}_{N+\frac{3}{2}}(\alpha v) J_{N+\frac{1}{2}}(v) P_{N}(t)\right) \\
& \text { and } S_{N}(v, \alpha)=\frac{\alpha v}{2} \sum_{\ell=N+1}^{\infty} \mathcal{B}_{\ell+\frac{1}{2}}(\alpha v)\left(J_{\ell-\frac{1}{2}}(v)-J_{\ell+\frac{3}{2}}(v)+\frac{1}{v} J_{\ell+\frac{1}{2}}(v)\right)\left(P_{\ell+1}(t)-P_{\ell-1}(t)\right)
\end{aligned}
$$

where $\mathcal{B}_{\nu}$ stands for the Bessel functions $J_{\nu}$ or $Y_{\nu}$.

Proof. First, let us recall a classic equality concerning Bessel functions:

$$
\mathcal{B}_{\nu-1}(v)+\mathcal{B}_{\nu+1}(v)=\frac{2 \nu}{v} \mathcal{B}_{\nu}(v)
$$


Let $S_{1}=\sum_{\ell=N+1}^{\infty}(2 \ell+1) \mathcal{B}_{\ell+\frac{1}{2}}(u) J_{\ell+\frac{1}{2}}(v) P_{\ell}(t)=u \sum_{\ell=N+1}^{\infty}\left(\mathcal{B}_{\ell+\frac{3}{2}}(u)+\mathcal{B}_{\ell-\frac{1}{2}}(u)\right) J_{\ell+\frac{1}{2}}(v) P_{\ell}(t)$. A discrete integration by part provides $S_{1}=S_{0}+R_{N}(v, \alpha)$, with $u=\alpha v, R_{N}(v, \alpha)$ given above and

$$
\begin{aligned}
S_{0} & =u \sum_{\ell=N+1}^{\infty} \mathcal{B}_{\ell+\frac{1}{2}}(u)\left(P_{\ell-1}(t) J_{\ell-\frac{1}{2}}(v)+P_{\ell+1}(t) J_{\ell+\frac{3}{2}}(v)\right) \\
& =\frac{u}{2} \sum_{\ell=N+1}^{\infty} \mathcal{B}_{\ell+\frac{1}{2}}(u)\left(\left(P_{\ell-1}(t)+P_{\ell+1}(t)\right)\left(J_{\ell-\frac{1}{2}}(v)+J_{\ell+\frac{3}{2}}(v)\right)+\left(P_{\ell-1}(t)-P_{\ell+1}(t)\right)\left(J_{\ell-\frac{1}{2}}(v)-J_{\ell+\frac{3}{2}}(v)\right)\right) .
\end{aligned}
$$

We use the recurrence formula for Legendre polynomials, ([10], p. 22): $P_{\ell-1}(t)+P_{\ell+1}(t)=2 t P_{\ell}(t)+\left(P_{\ell-1}(t)-\right.$ $\left.P_{\ell+1}(t)\right) /(2 \ell+1)$ and also $(37)$ with $\mathcal{B}_{\nu}(v)=J_{\ell+\frac{1}{2}}(v)$ to get $S_{0}=t \alpha \sum_{\ell=N+1}^{\infty}(2 \ell+1) J_{\ell+\frac{1}{2}}(v) \mathcal{B}_{\ell+\frac{1}{2}}(u) P_{\ell}(t)+$ $S_{N}(v, \alpha)$. The first series is exactly $t \alpha S_{1}$ and the result follows.

When $t= \pm 1, P_{\ell}(t)=1$ or $(-1)^{\ell}$ so that $P_{\ell+1}(t)=P_{\ell-1}(t)$ and the series $S_{N}$ vanishes, whence the corollary

Proposition 2.8. Let $v>0, \alpha>1$, then for all $N \geq 0$ the following equalities hold:

$$
\begin{aligned}
(\alpha-1) \sum_{\ell=N+1}^{\infty}(2 \ell+1) J_{\ell+\frac{1}{2}}(v) \mathcal{B}_{\ell+\frac{1}{2}}(\alpha v) & =\alpha v\left(\mathcal{B}_{N+\frac{3}{2}}(\alpha v) J_{N+\frac{1}{2}}(v)-\mathcal{B}_{N+\frac{1}{2}}(\alpha v) J_{N+\frac{3}{2}}(v)\right), \\
(\alpha+1) \sum_{\ell=N+1}^{\infty}(-1)^{\ell+N+1}(2 \ell+1) J_{\ell+\frac{1}{2}}(v) \mathcal{B}_{\ell+\frac{1}{2}}(\alpha v) & =\alpha v\left(\mathcal{B}_{N+\frac{3}{2}}(\alpha v) J_{N+\frac{1}{2}}(v)+\mathcal{B}_{N+\frac{1}{2}}(\alpha v) J_{N+\frac{3}{2}}(v)\right),
\end{aligned}
$$

where $\mathcal{B}_{\nu}$ stands for the Bessel functions $J_{\nu}$ or $Y_{\nu}$.

\section{Systematic ANALYSis OF THE UNIFORM ERROR}

Here we intend to establish some uniform estimates on the bounding series $e_{c}^{a b s}(u, v, L)$ defined in (10). In a first paragraph, we show the increase of $e_{c}^{a b s}(u, v, L)$ with respect to $v$. This result is of interest since it will allow us to consider the largest $v$ when uniform estimates will be required. The second, third and fourth paragraphs are devoted to the respective cases $L=N_{u}, L\left\langle N_{u}\right.$ and $\left.L\right\rangle N_{u}$. Numerical computations show the effectiveness of our estimates in the last case.

\subsection{Increase of $e_{c}^{a b s}(u, v, L)$ with respect to $v$}

In this part, we prove that it is possible to get rid of the constraint $0 \leq v \leq v_{\max }$ by showing that our estimate of $e_{c}^{a b s}$ is an increasing function of $v$, at least if $L+\frac{1}{2}$ is greater than $v_{\max }$. We do not know if we can do the same for $u$, showing that the estimate is a decreasing function of $u$, so we will work with the constraint $u=\alpha v \geq \alpha_{0} v$ from now on.

Proposition 3.1. Let $u, v_{\max }, u>v_{\max }$ be two positive numbers, and $L$ some integer such that $L+\frac{1}{2}>v_{\max }$; we have

$$
\sup _{v \leq v_{\max }} e_{c}^{a b s}(u, v, L) \leq \frac{\pi}{2}\left(\sqrt{\frac{u}{v_{\max }}}+\sqrt{\frac{v_{\max }}{u}}\right)\left(\sum_{\ell=L+1}^{\infty}(2 \ell+1) J_{\ell+\frac{1}{2}}\left(v_{\max }\right)\left|Y_{\ell+\frac{1}{2}}(u)\right|\right)=e_{c}^{a b s}\left(u, v_{\max }, L\right) .
$$


Proof. We assume that $u$ is fixed and greater than $\alpha_{0} v_{\max }, v$ smaller than $L+\frac{1}{2}$ and we consider (10) again

$$
e_{c}^{a b s}(u, v, L) \leq \frac{\pi}{2}\left(\sqrt{\frac{u}{v}}+\sqrt{\frac{v}{u}}\right)\left(\sum_{\ell=L+1}^{\infty}(2 \ell+1) J_{\ell+\frac{1}{2}}(v)\left|Y_{\ell+\frac{1}{2}}(u)\right|\right)
$$

using (26), we obtain

$$
e_{c}^{a b s}(u, v, L) \leq \frac{\pi}{2}\left(\sqrt{\frac{u}{v}}+\sqrt{\frac{v}{u}}\right)\left(\sum_{\ell=L+1}^{\infty}(2 \ell+1) J_{\ell+\frac{1}{2}}\left(v_{\max }\right)\left|Y_{\ell+\frac{1}{2}}(u)\right|\left(\frac{v}{v_{\max }}\right)^{\sqrt{\left(\ell+\frac{1}{2}\right)^{2}-v_{\max }^{2}}}\right) .
$$

Denote $\tau=\sqrt{\left(\ell+\frac{1}{2}\right)^{2}-v_{\max }^{2}}$. For all $\ell \geq L+1$,

$$
\left\{\begin{array}{l}
\sqrt{\frac{v}{u}}\left(\frac{v}{v_{\max }}\right)^{\tau}=\sqrt{\frac{v_{\max }}{u}}\left(\frac{v}{v_{\max }}\right)^{\tau+\frac{1}{2}} \leq \sqrt{\frac{v_{\max }}{u}} \\
\sqrt{\frac{u}{v}}\left(\frac{v}{v_{\max }}\right)^{\tau}=\sqrt{\frac{u}{v_{\max }}}\left(\frac{v}{v_{\max }}\right)^{\tau-\frac{1}{2}} \leq \sqrt{\frac{u}{v_{\max }}}
\end{array}\right.
$$

the latter equality coming from the fact that

$$
\sqrt{\left(L+\frac{3}{2}\right)^{2}-v_{\max }^{2}} \geq \sqrt{\left(v_{\max }+1\right)^{2}-v_{\max }^{2}}=\sqrt{2 v_{\max }+1}>1
$$

and (39) follows.

\subsection{Study of the error at the pivot value}

In this section, we focus on the particular case when $L=N_{u}$. The cornerstone of our estimates is the following corollary of Proposition (2.8).

Corollary 3.2. Let $u$ and $v$ two positive numbers $u=\alpha v>v$,

(i) If $0<u \leq N+\frac{5}{2}, 0<v \leq N+\frac{3}{2}$, then

$$
e_{c}^{a b s}(u, v, N) \leq-\frac{\pi}{2} \frac{\alpha+1}{\alpha-1} \alpha^{\frac{1}{2}} v\left(Y_{N+\frac{3}{2}}(u) J_{N+\frac{1}{2}}(v)-Y_{N+\frac{1}{2}}(u) J_{N+\frac{3}{2}}(v)\right) \text {. }
$$

(ii) If $0<u \leq N+\frac{3}{2}, 0<v \leq N+\frac{1}{2}$, then

$$
e_{c}^{a b s}(u, v, N) \leq-\frac{\pi}{2} \frac{\alpha+1}{\alpha-1} \alpha^{\frac{1}{2}} v Y_{N+\frac{3}{2}}(u) J_{N+\frac{1}{2}}(v)
$$

Proof. The first inequality is a direct consequence of Proposition (2.8). The second one comes from the fact that if $0<u \leq N+\frac{3}{2}$ and $0<v \leq N+\frac{1}{2}$, then the product $Y_{N+\frac{1}{2}}(u) J_{N+\frac{3}{2}}(v)$ is always negative, whereas $-Y_{N+\frac{3}{2}}(u) J_{N+\frac{1}{2}}(v)$ is positive.

This result will allow us to establish the

Proposition 3.3. Let $v_{\max }$ and $u=\alpha v_{\max }$ be two positive numbers. Assume that

$$
u>v_{\max }>\frac{1}{\alpha-1}
$$


let $N_{u}=\left[u-\frac{1}{2}\right]$, then we have

$$
e_{c}^{a b s}\left(u, v_{\max }, N_{u}\right) \leq \frac{3 C_{0}}{2} \sqrt{\frac{\pi}{2}} \frac{(\alpha+1) \alpha^{\frac{1}{6}}}{\alpha-1} \frac{v_{\max }^{\frac{2}{3}} \mathrm{e}^{-\left(N_{u}+\frac{1}{2}\right) F\left(\frac{v_{\max }}{N_{u}+\frac{1}{2}}\right)}}{\left(1-\left(\frac{v_{\max }}{N_{u}+\frac{1}{2}}\right)^{2}\right)^{\frac{1}{4}} \sqrt{N_{u}+\frac{1}{2}}}, \quad(F \text { given in }(24))
$$

with the possibility to turn 3 into 2 if $N_{u}$ is greater than 8.

Proof. Thanks to Hypothesis (42), we have

$$
v_{\max } \leq u-1<N_{u}+\frac{1}{2} \leq u<N_{u}+\frac{3}{2} \leq u+1, \text { with } N_{u}=\left[u-\frac{1}{2}\right],
$$

we can apply point $(i i)$ of Corollary 3.2 :

$$
e_{c}^{a b s}\left(u, v, N_{u}\right) \leq \frac{\pi}{2} \frac{\alpha+1}{\alpha-1} \sqrt{\alpha} v\left|Y_{N_{u}+\frac{3}{2}}(u)\right| J_{N_{u}+\frac{1}{2}}(v),
$$

where $v$ stands for $v_{\max }$ to lighten the notations. First we use

$$
Y_{N_{u}-\frac{1}{2}}(u)+Y_{N_{u}+\frac{3}{2}}(u)=\frac{2 N_{u}+1}{u} Y_{N_{u}+\frac{1}{2}}(u) .
$$

If $N_{u}$ is greater than 8 , then the function $Y_{N_{u}-\frac{1}{2}}(u)$ is negative, just like $Y_{N_{u}+\frac{1}{2}}(u)$ and $Y_{N_{u}+\frac{3}{2}}(u)$. Thus, by using (44) and the decrease of $\left|H^{(1)}(u)\right|$ with respect to $u$, and then by applying (20), we obtain

$$
\left|Y_{N_{u}+\frac{3}{2}}(u)\right| \leq \frac{2 N_{u}+1}{u}\left|Y_{N_{u}+\frac{1}{2}}(u)\right| \leq 2\left|H_{N_{u}+\frac{1}{2}}^{(1)}(u)\right| \leq 2\left|H_{u}(u)\right| \leq 2 \frac{C_{0}}{u^{\frac{1}{3}}}
$$

If $N_{u}$ is smaller than $8, Y_{N_{u}-\frac{1}{2}}(u)$ may be positive but we still can write

$$
\left|Y_{N_{u}+\frac{3}{2}}(u)\right| \leq \frac{2 N_{u}+1}{u}\left|Y_{N_{u}+\frac{1}{2}}(u)\right|+\left|Y_{N_{u}-\frac{1}{2}}(u)\right| \leq \frac{3 C_{0}}{u^{\frac{1}{3}}}
$$

The term $J_{N_{u}+\frac{1}{2}}(v)$ is estimated by (25). Since $v \leq N_{u}+\frac{1}{2}$,

$$
J_{N_{u}+\frac{1}{2}}(v) \leq \frac{\mathrm{e}^{-\left(N_{u}+\frac{1}{2}\right) F\left(\frac{v}{N_{u}+\frac{1}{2}}\right)}}{\left(1-\left(\frac{v}{N_{u}+\frac{1}{2}}\right)^{2}\right)^{\frac{1}{4}} \sqrt{2 \pi\left(N_{u}+\frac{1}{2}\right)}}
$$

We gather all those estimates, and (43) follows.

Our second estimate is uniform with respect to $u$ in $u>\alpha_{0} v_{\max }$. It is given below

Proposition 3.4. Let $\alpha_{0}$ be some number strictly greater than 1 .

$$
\text { If } v_{\max } \geq \frac{1}{\alpha_{0}-1} \text { then } \sup _{u \geq \alpha_{0} v_{\max }} e_{c}^{a b s}\left(u, v_{\max },\left[u-\frac{1}{2}\right]\right) \leq 3 \sqrt{\pi} C_{0} \frac{\alpha_{0}+1}{\alpha_{0}-1} \alpha_{0}^{\frac{2}{3}} v_{\max }^{\frac{1}{6}} \frac{\mathrm{e}^{-v_{\max } \alpha_{0} F\left(\frac{1}{\alpha_{0}}\right)}}{\left(1-\frac{1}{\alpha_{0}^{2}}\right)^{\frac{1}{4}}}
$$


First we use (27) in order to substitute $J_{N_{u}+\frac{3}{2}}(v)$ for $J_{N_{u}+\frac{1}{2}}(v)$ (with $v=v_{\max }$ ), before using (25). It follows that

$$
v J_{N_{u}+\frac{1}{2}}(v) \leq 2\left(N_{u}+\frac{3}{2}\right) J_{N_{u}+\frac{3}{2}}(v) \leq 2 \sqrt{N_{u}+\frac{3}{2}} \frac{\mathrm{e}^{-v \frac{F(x)}{x}}}{\left(1-x^{2}\right)^{\frac{1}{4}} \sqrt{2 \pi}}, \quad \text { with } x=\frac{v}{N_{u}+\frac{3}{2}},
$$

and since on the one hand $N_{u}+\frac{3}{2}$ is smaller than $u+1$ and on the other hand functions $x \rightarrow \frac{F(x)}{x}$ and $x \rightarrow\left(1-x^{2}\right)^{\frac{1}{4}}$ are decreasing over $] 0,1\left[\right.$, we can replace $x$ by $\frac{1}{\alpha}>\frac{v}{N_{u}+\frac{3}{2}}$. It yields

$$
v J_{N_{u}+\frac{1}{2}}(v) \leq \sqrt{\frac{2}{\pi}} u^{\frac{1}{2}} \frac{\mathrm{e}^{-v \alpha F\left(\frac{1}{\alpha}\right)}}{\left(1-\frac{1}{\alpha^{2}}\right)^{\frac{1}{4}}}\left(1+\frac{1}{u}\right)^{\frac{1}{2}}
$$

Lastly, the term $\left(1+\frac{1}{u}\right)$ is no trouble since, $v$ being supposed smaller than $u-1, u \geq \frac{\alpha}{\alpha-1}$ and $\sqrt{1+\frac{1}{u}}$ is bounded by $\sqrt{2-\frac{1}{\alpha}}$ and therefore by $\sqrt{2}$. Using (45) again, we obtain

$$
e_{c}^{a b s}\left(u, v, N_{u}\right) \leq \frac{\pi}{2} \frac{\alpha+1}{\alpha-1} \sqrt{\alpha} \frac{3 C_{0}}{(\alpha v)^{\frac{1}{3}}} \sqrt{\frac{2}{\pi}} \sqrt{\alpha v} \sqrt{2} \frac{\mathrm{e}^{-v \alpha F\left(\frac{1}{\alpha}\right)}}{\left(1-\frac{1}{\alpha^{2}}\right)^{\frac{1}{4}}}=3 \sqrt{\pi} C_{0} \frac{\alpha+1}{\alpha-1} \alpha^{\frac{2}{3}} v^{\frac{1}{6}} \frac{\mathrm{e}^{-v \alpha F\left(\frac{1}{\alpha}\right)}}{\left(1-\frac{1}{\alpha^{2}}\right)^{\frac{1}{4}}} .
$$

Assume $u \geq \alpha_{0} v$. A uniform estimate can be obtain easily: we have

$F\left(\frac{1}{\alpha}\right)=F\left(\frac{1}{\alpha_{0}}\right)+\int_{\frac{1}{\alpha_{0}}}^{\frac{1}{\alpha}} F^{\prime}(t) \mathrm{d} t=F\left(\frac{1}{\alpha_{0}}\right)+\int_{\frac{1}{\alpha}}^{\frac{1}{\alpha_{0}}} \frac{\sqrt{1-t^{2}}}{t} \mathrm{~d} t \quad \Rightarrow F\left(\frac{1}{\alpha}\right) \geq F\left(\frac{1}{\alpha_{0}}\right)+\log \left(\frac{\alpha}{\alpha_{0}}\right) \frac{1}{\alpha_{0}} \sqrt{\alpha_{0}^{2}-1}$

therefore

$$
e_{c}^{a b s}\left(u, v, N_{u}\right) \leq 3 \sqrt{\pi} C_{0} \frac{\alpha_{0}+1}{\alpha_{0}-1} \alpha_{0}^{\frac{2}{3}} v^{\frac{1}{6}}\left(\frac{\alpha_{0}}{\alpha}\right)^{-\frac{2}{3}+v \sqrt{\alpha_{0}^{2}-1}} \frac{\mathrm{e}^{-v \alpha_{0} F\left(\frac{1}{\alpha_{0}}\right)}}{\left(1-\frac{1}{\alpha_{0}^{2}}\right)^{\frac{1}{4}}} .
$$

Since $v \geq \frac{1}{\alpha-1} \geq \frac{1}{\alpha_{0}-1},-\frac{2}{3}+v \sqrt{\alpha_{0}^{2}-1}$ is positive, the result follows.

\subsection{Error estimate below the pivot value}

Here we assume that $L$ is strictly smaller than $N_{u}$. We also suppose that $v=v_{\max }$ is greater than $\frac{1}{\alpha-1}$ but not greater than $L+\frac{1}{2}$. We can split the remainder of the series, and display the term corresponding to the previous subsection, by writing

$$
e_{c}^{a b s}(u, v, L) \leq e_{c}^{1}(u, v, L)+e_{c}^{a b s}\left(u, v, N_{u}\right)
$$

with

$$
e_{c}^{1}(u, v, L)=\frac{\pi}{2} \frac{1+\alpha}{\sqrt{\alpha}}\left(\sum_{\ell=L+1}^{N_{u}}(2 \ell+1) J_{\ell+\frac{1}{2}}(v)\left|Y_{\ell+\frac{1}{2}}(u)\right|\right) .
$$

A first and very simple estimate consists in using the increase of $\left|H_{\nu}^{(1)}(u)\right|$ with respect to $\nu$, and upper bound (20)

$$
\left|Y_{\ell+\frac{1}{2}}(u)\right| \leq\left|H_{\ell+\frac{1}{2}}^{(1)}(u)\right| \leq\left|H_{N_{u}+\frac{1}{2}}^{(1)}(u)\right| \leq\left|H_{u}^{(1)}(u)\right| \leq \frac{C_{0}}{u^{\frac{1}{3}}} .
$$

Consequently

$$
e_{c}^{1}(u, v, L) \leq \frac{\pi}{2} \frac{1+\alpha}{\alpha^{\frac{5}{6}}} \frac{C_{0}}{v^{\frac{1}{3}}} \sum_{\ell=L+1}^{\infty}(2 \ell+1) J_{\ell+\frac{1}{2}}(v)
$$


and with estimate (32), it yields

$$
e_{c}^{1}(u, v, L) \leq \sqrt{\frac{\pi}{2}} C_{0} \frac{1+\alpha}{\alpha^{\frac{5}{6}}} v^{\frac{1}{6}} \sqrt{x} \frac{\mathrm{e}^{-v \frac{F(x)}{x}}}{\left(1-x^{2}\right)^{\frac{3}{4}}}, \quad x=\frac{v}{L+\frac{1}{2}}
$$

Gathering inequalities (43) and (47), we obtain:

Proposition 3.5. Let $v$ and $u=\alpha v$ be two positive numbers with $\frac{1}{\alpha-1}<v<u$, then for all $L$ such that $v \leq L+\frac{1}{2}<\left[\alpha v-\frac{1}{2}\right]+\frac{1}{2}$, we have

$$
\left\{\begin{array}{c}
e_{c}^{a b s}(u, v, L) \leq \sqrt{\frac{\pi}{2}} C_{0} \frac{1+\alpha}{\alpha}(\alpha v)^{\frac{1}{6}}\left(\sqrt{x} \frac{\mathrm{e}^{-v \frac{F(x)}{x}}}{\left(1-x^{2}\right)^{\frac{3}{4}}}+\frac{3 \alpha}{2(\alpha-1)} \sqrt{y} \frac{\mathrm{e}^{-v \frac{F(y)}{y}}}{\left(1-y^{2}\right)^{\frac{1}{4}}}\right) \\
x=\frac{v}{L+\frac{1}{2}}, \quad y=\frac{v}{\left[\alpha v-\frac{1}{2}\right]+\frac{1}{2}}, \quad \alpha=\frac{u}{v} .
\end{array}\right.
$$

This formula can be used in practice for fixed values of $u$ and $v$.

\subsection{Estimates beyond the pivot value}

The previous result shows that, for given values of $\alpha$ and $\epsilon$ and for $v$ large enough, we can obtain an error of the order of $\epsilon$ by choosing $L$ below the pivot value $N_{u}=\left[\alpha u-\frac{1}{2}\right]$. However, for small values of $v$ or $\epsilon$ or for $\alpha$ close to 1 , it is clear that $L$ must exceed that threshold to reach the required accuracy. In that case the problem of truncation occurs differently. Here we propose to give two estimates of the relative error, which allows us to understand its behaviour. An interesting consequence of those results is also to provide a method to compute quickly a truncation integer which guarantees an error smaller than $\epsilon$.

So we consider the case when $L$ is larger than $N_{u}=\left[u-\frac{1}{2}\right]$, and than $v-\frac{1}{2}$. Point (ii) of Corollary 3.2 can be applied, hence

$$
e_{c}^{a b s}(u, v, L) \leq-\frac{\pi}{2} \frac{\alpha+1}{\alpha-1} \alpha^{\frac{1}{2}} v Y_{L+\frac{3}{2}}(u) J_{L+\frac{1}{2}}(v)
$$

\subsubsection{A first estimate for $\alpha>\frac{\sqrt{5}}{2}$}

We use the following bound, which was established by Darve in ([11], pp. 120-134)

$$
\text { for all } \ell \geq \alpha v+\frac{7}{2}, \text { for all } \alpha>\frac{\sqrt{5}}{2}, \quad\left|\frac{(2 \ell+1) J_{\ell+\frac{1}{2}}(v) Y_{\ell+\frac{1}{2}}(\alpha v)}{(2 \ell-1) J_{\ell-\frac{1}{2}}(v) Y_{\ell-\frac{1}{2}}(\alpha v)}\right| \leq \frac{1}{\alpha}
$$

Since $\frac{Y_{L+\frac{3}{2}}(\alpha v)}{Y_{L+\frac{1}{2}}(\alpha v)}<\frac{2 L+1}{\alpha v}($ see $[2]$, p. 26$)$, it follows that for $L \geq\left[\alpha u-\frac{1}{2}\right]+4$

$$
e_{c}^{a b s}(u, v, L) \leq-\frac{\alpha+1}{\alpha-1} \pi\left(N+\frac{1}{2}\right) Y_{N+\frac{1}{2}}(\alpha v) J_{N+\frac{1}{2}}(v) \frac{1}{\alpha^{L+\frac{1}{2}-N}}, \quad N=\left[\alpha v-\frac{1}{2}\right]+4 .
$$

Actually this estimate turns out to be almost satisfactory, because it becomes efficient when $v$ is small (see Fig. 5), which is precisely the case when we must go beyond the pivot value.

Notice that the constraint on $\alpha$ has no consequence within the scope of the multilevel multipole method, because the minimum value of $\alpha$ is related to an oct-tree structure and a cube-splitting of the domain, and is always greater than $\frac{2}{\sqrt{3}}>\frac{\sqrt{5}}{2}$ (see $\left.[11]\right)$. 


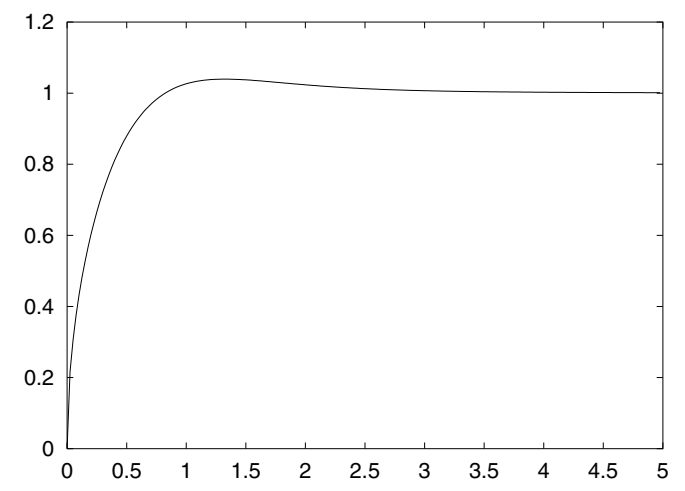

Figure 4. Graph of function $C(x)=2 \pi \sqrt{x} A i(x) B i(x)$.

\subsubsection{A second estimate}

Now we start from (49); we use (26) again, that is to say $\frac{v}{2} J_{L+\frac{1}{2}}(v) \leq\left(L+\frac{3}{2}\right) J_{L+\frac{3}{2}}(v)$, we denote $\nu=L+\frac{3}{2}$ and we write

$$
e_{c}^{a b s}(u, v, L) \leq-\pi \frac{\alpha+1}{\alpha-1} \alpha^{\frac{1}{2}} \nu Y_{\nu}(u) J_{\nu}(v)=\frac{\alpha+1}{\alpha-1} \alpha^{\frac{1}{2}}\left(-\pi \sqrt{\nu^{2}-u^{2}} Y_{\nu}(u) J_{\nu}(u)\right) \frac{\nu}{\sqrt{\nu^{2}-u^{2}}} \frac{J_{\nu}(v)}{J_{\nu}(u)} .
$$

Using the strong version of estimate (26), we obtain our second estimate

$$
e_{c}^{a b s}(u, v, L) \leq \frac{\alpha+1}{\alpha-1} \alpha^{\frac{1}{2}} C_{\max } \frac{\nu}{\sqrt{\nu^{2}-u^{2}}} \mathrm{e}^{-\nu\left(F\left(\frac{v}{\nu}\right)-F\left(\frac{u}{\nu}\right)\right)}, \quad \nu=L+\frac{3}{2}, \quad F \text { given in (24) }
$$

where

$$
C_{\max }=\sup _{u \leq \nu, \nu \geq \frac{1}{2}}\left(-\pi \sqrt{\nu^{2}-u^{2}} Y_{\nu}(u) J_{\nu}(u)\right) .
$$

The existence of the constant $C_{\max }$ comes from the following result:

Proposition 3.6. There exists a pure constant $C_{\max }$ greater than 1 and such that, if $\nu \geq \frac{1}{2}$ and $\nu \geq u$,

$$
-\pi \sqrt{\nu^{2}-u^{2}} Y_{\nu}(u) J_{\nu}(u) \leq C_{\max }, \quad\left(C_{\max } \leq 1.04 \text { (conjecture) }\right)
$$

Proof. This result is a direct consequence of the estimates on $\left|J_{\nu}(x)\right|$ and $\left|H_{\nu}^{(1)}(x)\right|$ that are given by Barcelo et al. in ([3], Lem. 1). More precisely, for some $\xi$ smaller than $1 / 2$, uniform estimates can be established in each of the 4 zones $\nu-\nu^{\frac{1}{3}} \leq u \leq \nu, \frac{\xi}{\nu} \leq u \leq \nu-\nu^{\frac{1}{3}}, 1<u<\frac{\xi}{\nu}$ and $0<u<1$. Then we just have to make estimates of the product (with a somewhat tedious though elementary calculation) to obtain the required result. A different and more direct proof could be derived from the following identity, given in [4],

$$
2 J_{\nu}(x) Y_{\nu}(x)=-\frac{4}{\pi} \int_{0}^{+\infty} J_{2 \nu}(2 x \cosh t) \mathrm{d} t .
$$

About the exact value of $C_{\max }$, the fact that it is larger than 1 comes from

$$
\lim _{\nu \rightarrow \infty}-\pi \nu Y_{\nu}(u) J_{\nu}(u)=1=\lim _{\nu \rightarrow \infty} \frac{\nu}{\sqrt{\nu^{2}-u^{2}}} \cdot
$$




$$
\alpha=\frac{2}{\sqrt{3}}:
$$
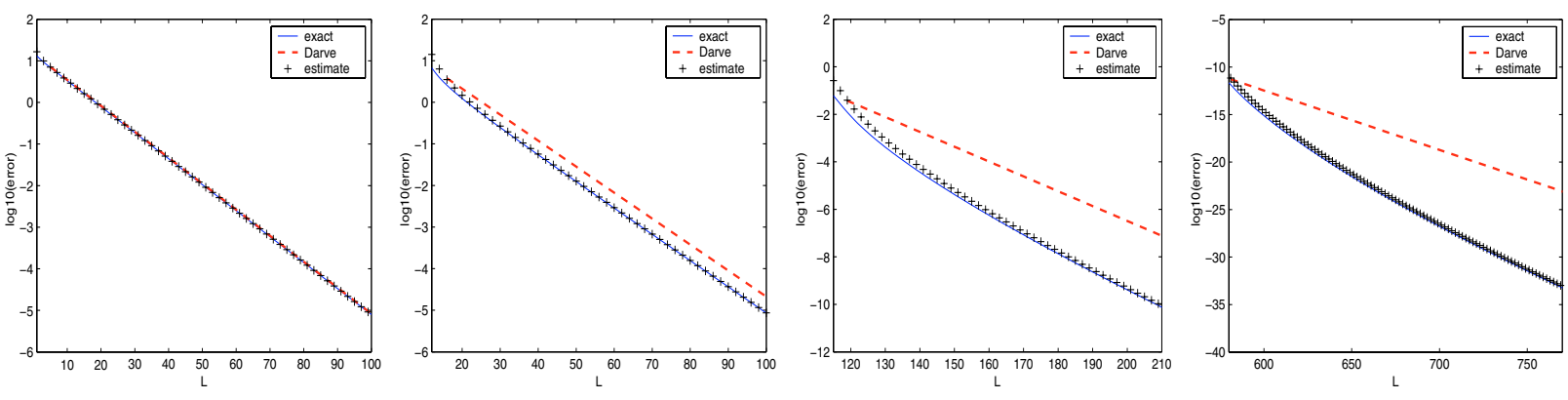

$\alpha=2$ :
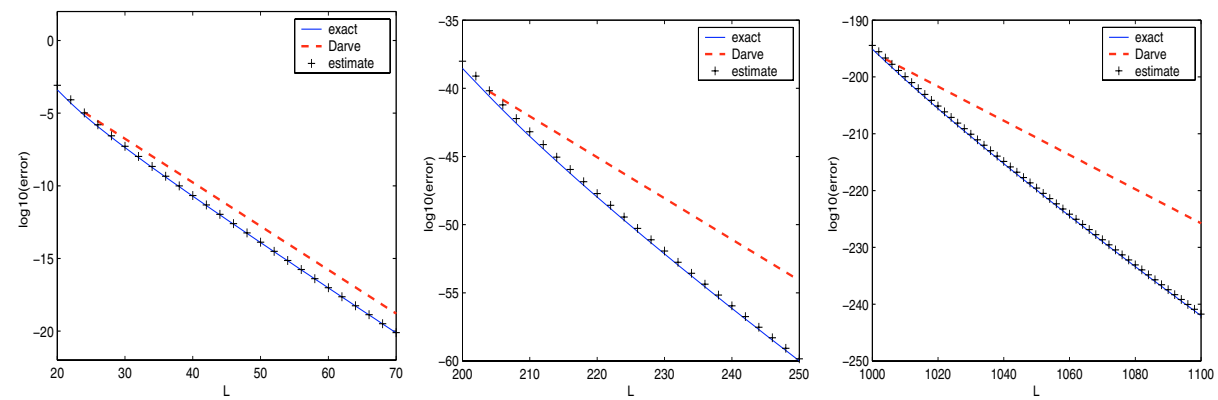

Figure 5. Comparison between true errors and estimates for $v=1,10,100,500$, and $\alpha=\frac{2}{\sqrt{3}}$ (top) and $v=10,100,500$ and $\alpha=2$ (bottom).

Moreover, if we use the asymptotic formulas given in [7], we can write for $s$ close to 0 and for $\xi(s)=$ $\left(\frac{3}{2}\left(\frac{1}{2} \log \frac{1+s}{1-s}-s\right)\right)^{\frac{2}{3}}$,

$$
\left\{\begin{array}{l}
J_{\mu}\left(\mu \sqrt{1-s^{2}}\right)=\frac{\sqrt{2}[\xi(s)]^{\frac{1}{4}} A i\left(\mu^{\frac{2}{3}} \xi(s)\right)}{\mu^{\frac{1}{3}} s^{\frac{1}{2}}}\left(1+O\left(\frac{1}{\mu}\right)\right) \\
Y_{\mu}\left(\mu \sqrt{1-s^{2}}\right)=-\frac{\sqrt{2}[\xi(s)]^{\frac{1}{4}} B i\left(\mu^{\frac{2}{3}} \xi(s)\right)}{\mu^{\frac{1}{3}} s^{\frac{1}{2}}}\left(1+O\left(\frac{1}{\mu}\right)\right)
\end{array}\right.
$$

$(A i(x)$ and $B i(x)$ are Airy functions [1]) from which we deduce

$$
\left\{\begin{array}{l}
-\pi \sqrt{\mu^{2}-u^{2}} J_{\mu}(u) Y_{\mu}(u) \sim 2 \pi \sqrt{x} A i(x) B i(x), \\
x=\xi(s) \mu^{\frac{2}{3}}=\left(\frac{3 \mu}{2}\left(\frac{1}{2} \log \frac{1+s}{1-s}-s\right)\right)^{\frac{2}{3}}=\left(\frac{3 \mu}{2} F\left(\frac{u}{\mu}\right)\right)^{\frac{2}{3}},
\end{array}\right.
$$

(we recognized the function $F$ defined in (24)). Since

$$
A i(x) \sim \frac{1}{2 \sqrt{\pi} x^{\frac{1}{4}}} \mathrm{e}^{-\frac{2}{3} x^{\frac{3}{2}}}, \quad B i(x) \sim \frac{1}{\sqrt{\pi} x^{\frac{1}{4}}} \mathrm{e}^{+\frac{2}{3} x^{\frac{3}{2}}}, x \rightarrow \infty
$$


we get

$$
\lim _{x \rightarrow \infty} C(x)=1, C(x)=2 \pi \sqrt{x} A i(x) B i(x) .
$$

However, function $C(x)$ is not monotonous for $x>0$, but increases from 0 to a maximum approximately equal to $1.0395 \ldots$, which is reached for $x \simeq 1.322$, and then decreases from that value and goes to 1 ( $c f$. Fig. 4). Hence we deduce that the constant $C_{\max }$ must be greater than $1.0395 \ldots$ In fact a large number of numerical experiments seem to point out that $C_{\max }<1.0395$ is sufficient.

\subsection{Use and test of our estimates}

From our results, we can propose a practical method of finding a truncation integer for a required relative accuracy $\epsilon$.

All the results we obtained may be gathered into the following algorithm: If $u$ and $v$ are fixed, $u=\alpha v$

- denote $N_{u}=\left[u-\frac{1}{2}\right]$ then estimate $e_{c}^{a b s}\left(u, v, N_{u}\right)$ using (43);

- if $e_{c}^{a b s}\left(u, v, N_{u}\right)>\epsilon$, use (51) or (50) in order to determine $L$;

- if $e_{c}^{a b s}\left(u, v, N_{u}\right)<\epsilon$, use (48) in order to determine $x$, then $L$.

In Figure 5 we show some error graphs for Gegenbauer, which allows us to see the behaviour of estimates beyond the pivot value $N_{u}$. The first four figures are drawn when $\alpha=\alpha_{0}=\frac{2}{\sqrt{3}}$, and when the value of $v$ is respectively $1,10,100$, and 500 . We make $L$ vary and we compare the true error with the upper bound we obtained as well as with Darve's one. In the following three figures, we take $\alpha=2$. We do not draw the case $v=1$ again, because nothing changes (all the curves nearly merge).

\section{Asymptotic error estimate for large values of $v$}

We intend to establish some uniform error estimates on sets of the following kind:

$$
\Omega_{\alpha_{0}, v_{\max }}=\left\{(v, u), u \geq \alpha_{0} v_{\max }, v \leq v_{\max }\right\}
$$

where $\alpha_{0}$ is a chosen positive number strictly greater than 1 , and $v_{\max }$ is a given positive number. More specifically, we are interested in the asymptotic expansion of the truncation integer $L\left(v_{\max }, \alpha_{0}, \epsilon\right)$ that gives an error $\epsilon$, uniform on $\Omega_{\alpha_{0}, v_{\max }}$, when $v_{\max }$ goes to infinity.

\subsection{Statement of the result}

Our result is stated in the following theorem.

Theorem 4.1. Let $\alpha_{0}$ be a number greater than 1, $v_{\max }$ a positive number, we consider the set

$$
\Omega=\left\{(v, u), u \geq \alpha_{0} v_{\max }, 0 \leq v \leq v_{\max }\right\}
$$

Let $\epsilon$ be a small positive number, we define $L_{a b s}\left(v_{\max }, \alpha_{0}, \epsilon\right)$ as $\left[\frac{v_{\max }}{x_{\epsilon}\left(v_{\max }\right)}-\frac{1}{2}\right]+1$ where $x_{\epsilon}\left(v_{\max }\right)$ is the unique solution of

$$
\Theta\left(x_{\epsilon}\left(v_{\max }\right)\right)=\frac{3}{2 v_{\max }} W\left(\frac{2\left(1+\alpha_{0}\right)^{2}}{3 \alpha_{0} \sqrt{\alpha_{0}^{2}-1}} \frac{v_{\max }}{\epsilon^{2}}\right),
$$

where $W(x)$ is Lambert's function (see (17)) and $\Theta(x)$ is the function defined in (31). Then we have

$$
\lim _{v_{\max } \rightarrow \infty} \sup _{u, v \in \Omega} e_{c}^{a b s}\left(u, v, L_{a b s}\left(v_{\max }, \alpha_{0}, \epsilon\right)\right) \leq \epsilon
$$


TABLE 2. Comparison between the truncation integers $L: L^{2}$ corresponds to the asymptotic formula (57), $L^{1}$ is the actual truncation integer.

\begin{tabular}{|c||c|c|c|c|c|}
\hline & $v=200$ & $v=1000$ & $v=10^{4}$ & $v=10^{5}$ & $v=10^{6}$ \\
\hline \hline$\alpha=\frac{2}{\sqrt{3}}$ & $L^{1}=222$ & $L^{1}=1039$ & $L^{1}=10093$ & $L^{1}=100217$ & $L^{1}=1000505$ \\
$\epsilon=10^{-2}$ & $L^{2}=221$ & $L^{2}=1039$ & $L^{2}=10093$ & $L^{2}=100217$ & $L^{2}=1000506$ \\
& $\Delta L=1$ & $\Delta L=0$ & $\Delta L=0$ & $\Delta L=0$ & $\Delta L=-1$ \\
\hline$\alpha=\frac{2}{\sqrt{3}}$ & $L^{1}=237$ & $L^{1}=1061$ & $L^{1}=10136$ & $L^{1}=100309$ & $L^{1}=1000697$ \\
$\epsilon=10^{-5}$ & $L^{2}=234$ & $L^{2}=1060$ & $L^{2}=10136$ & $L^{2}=100309$ & $L^{2}=1000698$ \\
& $\Delta L=3$ & $\Delta L=1$ & $\Delta L=0$ & $\Delta L=0$ & $\Delta L=-1$ \\
\hline$\alpha=2$ & $L^{1}=220$ & $L^{1}=1038$ & $L^{1}=10093$ & $L^{1}=100210$ & $L^{1}=1000490$ \\
$\epsilon=10^{-2}$ & $L^{2}=220$ & $L^{2}=1038$ & $L^{2}=10093$ & $L^{2}=100211$ & $L^{2}=1000491$ \\
& $\Delta L=0$ & $\Delta L=0$ & $\Delta L=0$ & $\Delta L=-1$ & $\Delta L=-1$ \\
\hline$\alpha=2$ & $L^{1}=233$ & $L^{1}=1059$ & $L^{1}=100137$ & $L^{1}=100303$ & $L^{1}=1000685$ \\
$\epsilon=10^{-5}$ & $L^{2}=233$ & $L^{2}=1059$ & $L^{2}=100137$ & $L^{2}=100304$ & $L^{2}=1000685$ \\
& $\Delta L=0$ & $\Delta L=0$ & $\Delta L=0$ & $\Delta L=-1$ & $\Delta L=0$ \\
\hline
\end{tabular}

Moreover, $L_{a b s}\left(v_{\max }, \alpha_{0}, \epsilon\right)$ satisfies to the following asymptotic expansion

$$
L_{a b s}\left(v_{\max }, \alpha_{0}, \epsilon\right)+\frac{1}{2}=v_{\max }+\frac{1}{2}\left(\frac{3}{2}\right)^{\frac{2}{3}}\left(v_{\max }\right)^{\frac{1}{3}} W^{\frac{2}{3}}\left(\frac{2\left(1+\alpha_{0}\right)^{2}}{3 \alpha_{0} \sqrt{\alpha_{0}^{2}-1}} \frac{v_{\max }}{\epsilon^{2}}\right)+\ldots
$$

Remark 4.2. As we said in the introduction, we focus on the "cosine" part of the error in the whole article. But it is obvious that, for the asymptotic analysis, $L$ is always placed in the zone between $v_{\max }$ and $\alpha_{0} v_{\max }$, where the behaviours of $J_{\ell+\frac{1}{2}}$ and $Y_{\ell+\frac{1}{2}}$ for $\ell \leq L$ are quite similar. So the asymptotic formula for the sine part would be the same. Actually, in all our proofs we always bounded $\left|Y_{\ell+\frac{1}{2}}\right|$ by $\left|H_{\ell+\frac{1}{2}}^{(1)}\right|$, which means not only that the result is the same for the total complex error, but also that this formula is optimal for the total complex error rather than for the cosine part. This will be true for all our asymptotic formulas.

We perform some tests on the quality of the asymptotic expansion (57): in Table 2, we give the values of $L^{1}=L^{\text {actual }}, L^{2}=L^{\text {ours }}$ and $\Delta L=L^{1}-L^{2}$ for different values of $v, \alpha_{0}$ and $\epsilon$ as a function of $v, L^{2}=L^{\text {ours }}$ being chosen according to (57) and $L^{1}=L^{\text {actual }}$ being the smallest truncation integer $L$ that experimentally satisfies to

$$
(u+v)\left(\sum_{\ell=L+1}^{\infty}(2 \ell+1)\left|j_{\ell}(v) h_{\ell}(u)\right|\right)<10^{-3}
$$

where $u=\alpha_{0} v, \alpha_{0}=\frac{2}{\sqrt{3}}$. The result is very good, even if we can notice that the asymptotic formula becomes valid only for rather large values of $v(>100)$.

\subsection{Proof of Theorem 4.1}

The proof is the most tedious and technical part of our article. Because of the term $(\alpha v)^{\frac{1}{6}}$, Estimate (48) is not appropriate for the asymptotic calculation. It means that using inequality (20) throughout the series is too rough. We must use the more accurate inequality (19) as far as possible. But we cannot use it everywhere, since the ratio $\frac{u}{\ell+\frac{1}{2}}$ approaches 1 just beyond the threshold $N_{u}$. That is why it is necessary to split the series more shrewdly, bounding some terms of the series thanks to (19) and the others with (20). 
Note that, according to Proposition 3.1, $e_{c}^{a b s}(u, v, L)$ is an increasing function of $v$. This allows us to consider only the case $v=v_{\max }$. To lighten the notations, we set $v=v_{\max }$ throughout the proof.

\subsubsection{Point 1 of the proof: splitting the series and first bounds}

Let $\lambda$ be some number such that

$$
1<\frac{L+\frac{1}{2}}{v}<\lambda<\alpha
$$

Setting $N_{\lambda v}=\left[\lambda v-\frac{1}{2}\right]$, we have $v<L+\frac{1}{2} \leq \lambda v<N_{\lambda v}+\frac{3}{2}$. We split the series in (10) into four parts

$$
e_{c}^{a b s}(u, v, L) \leq \sum_{p=1}^{3} e_{c}^{p}(u, v)+e_{c}^{a b s}\left(u, v, N_{u}\right) \text { with }\left\{\begin{aligned}
e_{c}^{1}(u, v) & =\frac{\pi}{2} \frac{1+\alpha}{\sqrt{\alpha}}\left(\sum_{\ell=L+1}^{N_{\lambda v}}(2 \ell+1) J_{\ell+\frac{1}{2}}(v)\left|Y_{\ell+\frac{1}{2}}(u)\right|\right) \\
e_{c}^{2}(u, v) & =\frac{\pi}{2} \frac{1+\alpha}{\sqrt{\alpha}} 2\left(N_{\lambda v}+\frac{3}{2}\right) J_{N_{\lambda v}+\frac{3}{2}}(v)\left|Y_{N_{\lambda v}+\frac{3}{2}}(u)\right| \\
e_{c}^{3}(u, v) & =\frac{\pi}{2} \frac{1+\alpha}{\sqrt{\alpha}}\left(\sum_{\ell=N_{\lambda}+2}^{N_{u}}(2 \ell+1) J_{\ell+\frac{1}{2}}(v)\left|Y_{\ell+\frac{1}{2}}(u)\right|\right),
\end{aligned}\right.
$$

(for technical reasons we separate the term corresponding to $\ell=N_{\lambda v}+1$ from those corresponding to $\left.\ell>N_{\lambda v}+1\right)$.

For $e_{c}^{2}(u, v)$ and $e_{c}^{3}(u, v)$, we still use the same bound of $\left|Y_{\ell+\frac{1}{2}}(u)\right|$, that is to say $C_{0} u^{-\frac{1}{3}}$, whereas $e_{c}^{1}(u, v)$ can be bounded by using $\mid Y_{\ell+\frac{1}{2}}\left(u|\leq| H_{\ell+\frac{1}{2}}^{(1)}(u)|\leq| H_{\lambda v}^{(1)}(u) \mid\right.$ and inequality (19). After reordering the terms, it follows

$$
\left\{\begin{aligned}
& e_{c}^{1}(u, v) \leq \frac{1+\alpha}{\sqrt{\alpha}\left(\alpha^{2}-1\right)^{\frac{1}{4}}}\left(\left(\frac{\alpha^{2}-1}{\alpha^{2}-\lambda^{2}}\right)^{\frac{1}{4}} \sqrt{\frac{\pi}{2 v}} \sum_{\ell=L+1}^{N_{\lambda v}}(2 \ell+1) J_{\ell+\frac{1}{2}}(v)\right) \\
& e_{c}^{2}(u, v) \leq \frac{1+\alpha}{\alpha}\left((\alpha v)^{\frac{1}{6}} C_{0} \sqrt{\frac{\pi}{2}} 2\left(N_{\lambda v}+\frac{3}{2}\right) \sqrt{\frac{\pi}{2 v}} J_{N_{\lambda v}+\frac{3}{2}}(v)\right) \\
& e_{c}^{3}(u, v) \leq \frac{1+\alpha}{\alpha}\left((\alpha v)^{\frac{1}{6}} C_{0} \sqrt{\frac{\pi}{2}} \sqrt{\frac{\pi}{2 v}} \sum_{\ell=N_{\lambda v}+2}^{N_{u}}(2 \ell+1) J_{\ell+\frac{1}{2}}(v) \mid\right) .
\end{aligned}\right.
$$

From these formulas, we build other estimates by using both bounds (25) for the Bessel functions and (32) for the remainder of the Jacobi-Anger series. We obtain

$$
\left\{\begin{aligned}
& e_{c}^{1}(u, v) \leq \frac{1+\alpha}{\sqrt{\alpha}\left(\alpha^{2}-1\right)^{\frac{1}{4}}}\left(\left(\frac{\alpha^{2}-1}{\alpha^{2}-\lambda^{2}}\right)^{\frac{1}{4}} \sqrt{x} \frac{\mathrm{e}^{-v \frac{F(x)}{x}}}{\left(1-x^{2}\right)^{\frac{3}{4}}}\right) \\
& e_{c}^{2}(u, v) \leq \frac{1+\alpha}{\alpha}\left((\alpha v)^{\frac{1}{6}} C_{0} \sqrt{\frac{\pi}{2}} \frac{\mathrm{e}^{-v \frac{F\left(x_{\lambda}\right)}{x_{\lambda}}}}{\sqrt{x_{\lambda}}\left(1-x_{\lambda}^{2}\right)^{\frac{1}{4}}}\right) \\
& e_{c}^{3}(u, v) \leq \frac{1+\alpha}{\alpha}\left((\alpha v)^{\frac{1}{6}} C_{0} \sqrt{\frac{\pi}{2}} \sqrt{x_{\lambda}} \frac{\mathrm{e}^{-v \frac{F\left(x_{\lambda}\right)}{x_{\lambda}}}}{\left(1-x_{\lambda}^{2}\right)^{\frac{3}{4}}}\right),
\end{aligned}\right.
$$

with

$$
x=\frac{v}{L+\frac{1}{2}}, x_{\lambda}=\frac{v}{N_{\lambda v}+\frac{3}{2}} \leq \frac{1}{\lambda} .
$$

Our purpose is to show that the dominant term is $e_{c}^{1}$ for large $v$. We will choose $x$ (i.e. L) such that $e_{c}^{1}$ is asymptotically $\epsilon$ in a first paragraph. The best $\lambda$ is the one which minimizes $\left(e_{c}^{1}+e_{c}^{2}+e_{c}^{3}\right)(u, v)$, but that minimum is very difficult to reach analytically. Here we will take a more practical approach, by choosing a $\lambda$, which is certainly not optimal, but which allows us to obtain an acceptable asymptotic error estimate. 
The difficult point is to take $\lambda$ close enough to 1 in order to minimize the constant in the estimate of $e_{c}^{1}$, but not too close, so that $e_{c}^{2}$ and $e_{c}^{3}$ keep being negligible with respect to $e_{c}^{1}$. This choice will be done (once $x$ will have been fixed) in a second paragraph.

\subsubsection{Point 2 of the proof: choosing $L$ or $x$ and further bounds}

We still assume that $u=\alpha v \geq \alpha_{0} v$ and that $\epsilon$ is positive real number. First we choose $L$ such that $e_{c}^{1}(u, v)$ is of the order of $\epsilon$ for $\lambda=1$ and for the most constraining value of $\alpha$, that is to say $\alpha_{0}$ since

$$
K\left(\alpha_{0}\right)=\frac{1+\alpha_{0}}{\sqrt{\alpha_{0}}\left(\alpha_{0}^{2}-1\right)^{\frac{1}{4}}}>\frac{1+\alpha}{\sqrt{\alpha}\left(\alpha^{2}-1\right)^{\frac{1}{4}}}, \text { when } \alpha>\alpha_{0}
$$

As $\frac{F(x)}{x}>\frac{1}{3} \Theta(x), \Theta(x)$ being the strictly decreasing function given in (31), it follows that

$$
e_{c}^{1}(u, v) \leq \frac{1+\alpha_{0}}{\sqrt{\alpha_{0}}\left(\alpha_{0}^{2}-1\right)^{\frac{1}{4}}}\left(\frac{\alpha^{2}-1}{\alpha^{2}-\lambda^{2}}\right)^{\frac{1}{4}} \frac{\mathrm{e}^{-\frac{v}{3} \Theta(x)}}{\sqrt{\Theta(x)}}
$$

Let $x_{\epsilon}(v)$ be given by

$$
\Theta\left(x^{\epsilon}(v)\right)=\frac{3}{2 v} W\left(\frac{2 K^{2}\left(\alpha_{0}\right) v}{3 \epsilon^{2}}\right) \Longleftrightarrow \frac{\mathrm{e}^{-\frac{v}{3} \Theta\left(x^{\epsilon}(v)\right)}}{\sqrt{\Theta\left(x^{\epsilon}(v)\right)}}=\frac{\epsilon}{K\left(\alpha_{0}\right)},
$$

where $W(x)$ is the Lambert function defined in (17). Since $x^{\epsilon}(v)$ is not necessarily in the form $\frac{v}{k+\frac{1}{2}}$ with $k$ an integer, we take

$$
x=\frac{v}{L+\frac{1}{2}}=\frac{v}{\left[\frac{v}{x_{\epsilon}(v)}-\frac{1}{2}\right]+\frac{1}{2}}, \quad\left(\frac{1}{x_{\epsilon}(v)}-\frac{1}{v} \leq \frac{1}{x} \leq \frac{1}{x_{\epsilon}(v)}\right) .
$$

We get the bound

$$
e_{c}^{1}(u, v) \leq T(\lambda, \alpha) \epsilon, \text { with } T(\lambda, \alpha)=\left(\frac{\alpha^{2}-1}{\alpha^{2}-\lambda^{2}}\right)^{\frac{1}{4}} .
$$

Since $\lambda / \alpha<1$ and $\alpha \geq \alpha_{0}, T(\lambda, \alpha)$ satisfies to (cf. Def. (31) of function $\Theta$ )

$$
\frac{1}{T^{4}(\lambda, \alpha)}=1-\frac{\lambda^{2}}{\alpha^{2}} \frac{1-\frac{1}{\lambda^{2}}}{1-\frac{1}{\alpha^{2}}}=1-\frac{\lambda^{\frac{4}{3}} \Theta\left(\frac{1}{\lambda}\right)^{\frac{2}{3}}}{\alpha^{2}\left(1-\frac{1}{\alpha^{2}}\right)} \geq 1-\left(\frac{\Theta\left(\frac{1}{\lambda}\right)}{\alpha}\right)^{\frac{2}{3}} \frac{1}{\left(1-\frac{1}{\alpha_{0}^{2}}\right)} .
$$

Therefore, if $\frac{\Theta\left(\frac{1}{\lambda}\right)}{\alpha}$ is small enough

$$
e_{c}^{1}(u, v) \leq\left(1-\left(\frac{\Theta\left(\frac{1}{\lambda}\right)}{\alpha}\right)^{\frac{2}{3}}\left(1-\frac{1}{\alpha_{0}^{2}}\right)^{-1}\right)^{-\frac{1}{4}} \epsilon
$$

Now we focus on the terms $e_{c}^{2}(u, v)$ and $e_{c}^{3}(u, v)$, and we consider (58). For $e_{c}^{3}(u, v)$ we use (24), that is to say $F\left(x_{\lambda}\right) x_{\lambda}{ }^{-1} \geq \frac{1}{3} \Theta\left(x_{\lambda}\right) \geq \frac{1}{3} \Theta\left(\frac{1}{\lambda}\right)$, the latter bound coming from the fact that $x_{\lambda} \leq \frac{1}{\lambda}$. For $e_{c}^{2}(u, v)$, we use a more accurate lower bound by considering two terms in the development of the function $F(x)$ at point $x=1$

$$
\frac{F\left(x_{\lambda}\right)}{x_{\lambda}} \geq \frac{1}{3} \Theta\left(\frac{1}{\lambda}\right)+\frac{1}{5} \frac{\left(1-x_{\lambda}^{2}\right)^{\frac{5}{2}}}{x_{\lambda}},
$$


then we use the rather rough bounds

$$
\frac{v^{\frac{1}{5}}\left(1-x_{\lambda}^{2}\right)^{\frac{1}{2}}}{x_{\lambda}^{\frac{1}{5}}} \mathrm{e}^{-\frac{v}{5} \frac{\left(1-x_{\lambda}{ }^{2}\right)^{\frac{5}{2}}}{x_{\lambda}}} \leq \sup _{t>0}\left(t \mathrm{e}^{-t}\right)^{\frac{1}{5}}=\frac{1}{\mathrm{e}^{\frac{1}{5}}}, \quad \text { and also } x_{\lambda}>\frac{1}{2 \alpha},
$$

the latter inequality coming from

$$
x_{\lambda}=\frac{v}{N_{\lambda v}+\frac{3}{2}} \geq \frac{v}{\lambda v+1}=\frac{1}{\alpha} \frac{\alpha v}{\lambda v+1} \geq \frac{1}{\alpha} \frac{\alpha v}{\alpha v+1}
$$

and $\frac{\alpha v}{\alpha v+1}$ is greater than $\frac{1}{2}$ (for instance) if $\alpha_{0} v=u$ is greater than 1 . Then it follows

$$
e_{c}^{2}(u, v) \leq \frac{1+\alpha_{0}}{\alpha_{0}}(\alpha v)^{\frac{1}{6}} C_{0} \sqrt{\frac{\pi}{2}} \frac{(2 \alpha)^{\frac{4}{5}}}{(e v)^{\frac{1}{5}}} \frac{\mathrm{e}^{-\frac{v}{3} \Theta\left(\frac{1}{\lambda}\right)}}{\sqrt{\Theta\left(\frac{1}{\lambda}\right)}}, \quad e_{c}^{3}(u, v) \leq \frac{1+\alpha_{0}}{\alpha_{0}}(\alpha v)^{\frac{1}{6}} C_{0} \sqrt{\frac{\pi}{2}} \frac{\mathrm{e}^{-\frac{v}{3} \Theta\left(\frac{1}{\lambda}\right)}}{\sqrt{\Theta\left(\frac{1}{\lambda}\right)}}
$$

Whence

$$
\left(e_{c}^{2}+e_{c}^{3}\right)(u, v) \leq C_{0} \sqrt{\frac{\pi}{2}} \frac{1+\alpha_{0}}{\alpha_{0}}(\alpha v)^{\frac{1}{6}} \frac{\mathrm{e}^{-\frac{v}{3} \Theta\left(\frac{1}{\lambda}\right)}}{\sqrt{\Theta\left(\frac{1}{\lambda}\right)}}\left(1+\frac{(2 \alpha)^{\frac{4}{5}}}{(e v)^{\frac{1}{5}}}\right)
$$

\subsubsection{Point 3 of the proof: choosing $\lambda$}

As we said before, in order to have an error asymptotically equal to $\epsilon$, we must choose $\lambda$ large enough for the term $e_{c}^{2}(u, v)+e_{c}^{3}(u, v)$ to be negligible, but small enough for $\frac{\Theta\left(\frac{1}{\lambda}\right)}{\alpha}$ to go uniformly to 0 when $v$ goes to infinity, $\alpha$ staying beyond $\alpha_{0}$.

The function $\xi \rightarrow \frac{3}{2 \xi} W\left(\frac{2 \xi^{2}}{3 \epsilon^{2}}\right)$ goes to 0 when $\xi$ goes to infinity. Therefore there exists a number $U_{\epsilon, \alpha_{0}}$ such that

$$
\forall u>U_{\epsilon, \alpha_{0}}, \quad \frac{3}{2 u} W\left(\frac{2 u^{2}}{3 \epsilon^{2}}\right)<\frac{1}{2 \sqrt{2}}\left(1-\frac{1}{\alpha_{0}^{2}}\right)^{\frac{3}{2}} .
$$

We choose $\lambda=\lambda^{\epsilon}(v, \alpha)$ as the solution of

$$
\Theta\left(\frac{1}{\lambda}\right)=2 \sqrt{2} \frac{3}{2 v} W\left(\frac{2(\alpha v)^{2}}{3 \epsilon^{2}}\right) .
$$

One can verify that, if $\alpha v$ is greater than $U_{\epsilon, \alpha_{0}}$,

$$
\Theta\left(\frac{1}{\lambda^{\epsilon}(v, \alpha)}\right)<\alpha\left(1-\frac{1}{\alpha_{0}^{2}}\right)^{\frac{3}{2}} \leq \Theta\left(\frac{1}{\alpha}\right)
$$

and, if $v$ is large, or more precisely if

$$
\alpha_{0}^{2} v \geq \frac{\left(1+\alpha_{0}\right)^{2}}{\alpha_{0}\left(\alpha_{0}^{2}-1\right)^{\frac{1}{2}}}
$$

which holds if $\alpha_{0} v>\frac{\alpha_{0}}{\alpha_{0}-1}$, we get

$$
2 \sqrt{2} \Theta\left(x^{\epsilon}(v)\right)=2 \sqrt{2} \frac{3}{2 v} W\left(\frac{2 K^{2}\left(\alpha_{0}\right) v}{3 \epsilon^{2}}\right)<2 \sqrt{2} \frac{3}{2 v} W\left(\frac{2\left(\alpha_{0} v\right)^{2}}{3 \epsilon^{2}}\right) \leq \Theta\left(\frac{1}{\lambda^{\epsilon}(v, \alpha)}\right) .
$$

Thus we have

$$
\Theta\left(x^{\epsilon}(v)\right)<2 \sqrt{2} \Theta\left(x^{\epsilon}(v)\right)<\Theta\left(\frac{1}{\lambda^{\epsilon}(v, \alpha)}\right)<\Theta\left(\frac{1}{\alpha}\right) .
$$




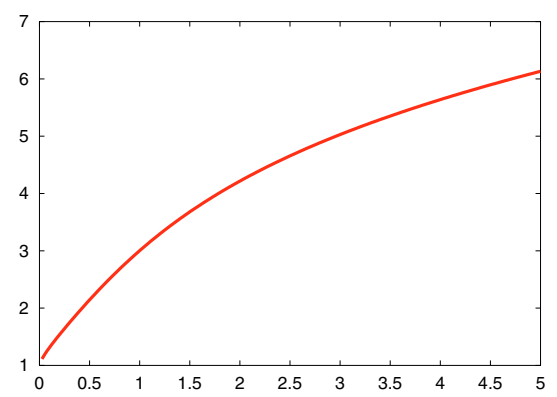

Figure 6. Graph of Function $\theta \rightarrow\left(\frac{2}{\Theta^{-1}(2 \sqrt{2} \theta)}-\frac{2}{\Theta^{-1}(\theta)}\right) \theta^{-\frac{2}{3}}$.

Assume that it is possible to slip $\Theta(x)$ with $x$ given in (60) between the first two terms of the series of inequalities above. Then the constraint $1 / x<\lambda^{\epsilon}(v, \alpha)<\alpha$ would be satisfied $(\Theta(x)$ is a strictly decreasing function of $x)$ and our choice of $\lambda$ valid. We prove that it is the case at least when $v$ is large enough: we use the abstruse property (see Fig. 6)

$$
\forall \theta \geq 0, \quad \frac{1}{\Theta^{-1}(2 \sqrt{2} \theta)}-\frac{1}{\Theta^{-1}(\theta)} \geq \frac{1}{2} \theta^{\frac{2}{3}}
$$

and get that if

$$
\Theta\left(y_{\epsilon}(v)\right)=2 \sqrt{2} \Theta\left(x_{\epsilon}(v)\right) \text { and } \Delta L=\frac{v}{y^{\epsilon}(v)}-\frac{v}{x^{\epsilon}(v)}
$$

then $\Delta L \geq 1$ when $v \Theta\left(x_{\epsilon}(v)\right)^{\frac{2}{3}} \geq 2$ and a fortiori (since $\alpha>\alpha_{0}$ ) when

$$
v W^{2}\left(\frac{2 K^{2}\left(\alpha_{0}\right) v}{3 \epsilon^{2}}\right) \geq \frac{32}{9}
$$

and the constraint $\frac{1}{x}<\lambda^{\epsilon}(v, \alpha)<\alpha$ is finally satisfied.

\subsubsection{Last point of the proof: final bounds}

With our choice of $\lambda$, and since

$$
\Theta\left(\frac{1}{\lambda}\right)=2 \sqrt{2} \frac{3}{2 v} W\left(\frac{2(\alpha v)^{2}}{3 \epsilon^{2}}\right) \Leftrightarrow \quad \frac{\mathrm{e}^{-\frac{v}{3} \Theta\left(\frac{1}{\lambda}\right)}}{\sqrt{\Theta\left(\frac{1}{\lambda}\right)}} \leq \frac{\epsilon}{2 \alpha \sqrt{2 v}}
$$

the estimate on $\left(e_{c}^{2}+e_{c}^{3}\right)(u, v)$ gives

$$
\left(e_{c}^{2}+e_{c}^{3}\right)(u, v) \leq C_{0} \sqrt{\frac{\pi}{2}} \frac{1+\alpha_{0}}{\alpha_{0}}(\alpha v)^{\frac{1}{6}} \frac{\epsilon}{2 \alpha \sqrt{2 v}}\left(1+\frac{(2 \alpha)^{\frac{4}{5}}}{(e v)^{\frac{1}{5}}}\right)
$$

and finally

$$
\left(e_{c}^{2}+e_{c}^{3}\right)(u, v) \leq C_{0} \sqrt{\frac{\pi}{2}} \frac{1+\alpha_{0}}{\alpha_{0}}\left(\frac{1}{\alpha_{0}^{\frac{5}{6}}}+\frac{2^{\frac{4}{5}}}{(e v)^{\frac{1}{5}} \alpha_{0}^{\frac{1}{30}}}\right) \frac{\epsilon}{2 \sqrt{2} v^{\frac{1}{3}}} .
$$


We have almost finished, still we just have to estimate the term $e_{c}^{a b s}\left(u, v, N_{u}\right)$. For that purpose, we consider (46) again. For all $u$ greater than $\alpha_{0} v, U_{\epsilon, \alpha_{0}}$ and $\frac{\alpha_{0}}{1-\alpha_{0}}$

$$
\begin{aligned}
& e_{c}^{a b s}\left(u, v, N_{u}\right) \leq 3 \sqrt{\pi} C_{0} \frac{\alpha_{0}+1}{\alpha_{0}-1} \alpha_{0}^{\frac{2}{3}} v^{\frac{1}{6}} \frac{\mathrm{e}^{-\frac{v}{3} \Theta\left(\frac{1}{\alpha_{0}}\right)}}{\left(1-\frac{1}{\alpha_{0}^{2}}\right)^{\frac{1}{4}}}=3 \sqrt{\pi} C_{0} \frac{\alpha_{0}+1}{\alpha_{0}-1}\left(1-\frac{1}{\alpha_{0}^{2}}\right)^{\frac{1}{2}} \alpha_{0}^{\frac{1}{6}} v^{\frac{1}{6}} \frac{\mathrm{e}^{-\frac{v}{3} \Theta\left(\frac{1}{\alpha_{0}}\right)}}{\sqrt{\Theta\left(\frac{1}{\alpha_{0}}\right)}} \Rightarrow \\
& e_{c}^{a b s}\left(u, v, N_{u}\right) \leq 3 \sqrt{\pi} C_{0} \frac{\alpha_{0}+1}{\alpha_{0}-1}\left(1-\frac{1}{\alpha_{0}^{2}}\right)^{\frac{1}{2}} \alpha_{0}^{\frac{1}{6}} v^{\frac{1}{6}} \frac{\epsilon}{\alpha_{0} 2 \sqrt{2 v}},\left(\operatorname{use} \Theta\left(\frac{1}{\alpha_{0}}\right) \geq \Theta\left(\frac{1}{\lambda^{\epsilon}\left(v, \alpha_{0}\right)}\right) \text { then }(63)\right)
\end{aligned}
$$

and finally

$$
e_{c}^{a b s}\left(u, v, N_{u}\right) \leq 3 \sqrt{\pi} C_{0} \frac{\alpha_{0}+1}{\alpha_{0}-1}\left(1-\frac{1}{\alpha_{0}^{2}}\right)^{\frac{1}{2}} \frac{\epsilon}{\alpha_{0}^{\frac{5}{6}} 2 \sqrt{2} v^{\frac{1}{3}}} .
$$

We gather estimates $(62),(64)$ and (65). We recall that $L$ is chosen equal to $L_{a b s}\left(v, \alpha_{0}, \epsilon\right)=\frac{v}{x^{\epsilon}(v)}-\frac{1}{2}, x^{\epsilon}(v)$ given by (59). We assume that $u$ is greater than $\alpha_{0} v$ and $v$ is large enough (with respect to some functions depending only on $\alpha_{0}$ and $\epsilon$ ), to satisfy all the hypotheses we have made. It follows that

$\forall \alpha_{0}>1$, there exist some $c_{1}, c_{2}, c_{3}$ depending on $\alpha_{0}$ only such that when $v$ is large enough

$$
\begin{gathered}
e_{c}^{a b s}\left(u, v, L_{a b s}\left(v, \alpha_{0}, \epsilon\right)\right) \leq F\left(\alpha_{0}, v\right) \epsilon, \text { with } \\
F\left(\alpha_{0}, v\right)=\frac{1}{\left(1-c_{1} \zeta\left(\alpha_{0} v\right)\right)^{\frac{1}{4}}}+\left(c_{2}+\frac{c_{3}}{v^{\frac{1}{5}}}\right) \frac{1}{v^{\frac{1}{3}}} \text { and } \zeta\left(\alpha_{0} v\right)=\left(\frac{3}{2 \alpha_{0} v} W\left(\frac{2\left(\alpha_{0} v\right)^{2}}{3 \epsilon^{2}}\right)\right)^{\frac{2}{3}} .
\end{gathered}
$$

Now we have got all the elements to conclude: when $v=v_{\max }$ goes to infinity, $F\left(\alpha_{0}, v_{\max }\right)$ goes to 1 and the error is asymptotically bounded by $\epsilon$. Because of the increase of the error $e_{c}^{a b s}$ with respect to $v$, the theorem follows.

\section{ANALYSIS FOR $\hat{\mathbf{u}} \cdot \hat{\mathbf{v}}$ GIVEN}

Here we consider the case when directions $\hat{\mathbf{u}}$ and $\hat{\mathbf{v}}$ are fixed as well as $\alpha$, and $u$ and $v$ vary. Our goal is to better understand the dependency of the error on the relative configuration of vectors $\vec{u}$ and $\vec{v}$.

\subsection{The case $\hat{\mathbf{u}} \cdot \hat{\mathbf{v}}=-1$}

Now we focus on the initial series $e_{c}(\vec{u}, \vec{v}, L)$ rather than the one with absolute values. By replacing $P_{\ell}(\hat{u} \cdot \hat{v})$ by its very value, let $(-1)^{\ell}$, we obtain

$$
e_{c}(\vec{u}, \vec{v}, L)=|\vec{u}+\vec{v}|\left|\sum_{\ell=L+1}^{\infty}(2 l+1) y_{\ell}(u) j_{\ell}(v)\right| .
$$

In view of Proposition 2.8, we can give an explicit form for this series, and, after having noticed that $\vec{u}+\vec{v}=$ $(\alpha-1) \vec{v}$, it follows that

$$
e_{c}(\vec{u}, \vec{v}, L)=\frac{\pi}{2} \sqrt{u v}\left|Y_{L+\frac{3}{2}}(u) J_{L+\frac{1}{2}}(v)-Y_{L+\frac{1}{2}}(u) J_{L+\frac{3}{2}}(v)\right|, \quad \text { when } \hat{u} \cdot \hat{v}=-1
$$

and,

$$
\left\{\begin{aligned}
e_{c}(\vec{u}, \vec{v}, L) \leq & \left(\sqrt{\frac{\pi v}{2}} J_{L+\frac{1}{2}}(v)\right)\left(\sqrt{\frac{\pi u}{2}}\left|H_{L+\frac{3}{2}}^{(1)}(u)-H_{L+\frac{1}{2}}^{(1)}(u)\right|\right) \\
& +\left(\sqrt{\frac{\pi v}{2}}\left(J_{L+\frac{3}{2}}(v)-J_{L+\frac{1}{2}}(v)\right)\left(\sqrt{\frac{\pi u}{2}}\left|H_{L+\frac{1}{2}}^{(1)}(u)\right|\right) .\right.
\end{aligned}\right.
$$


Assume that $v$ is large and that $L-v$ does not increase faster than $C v^{\delta}$ for some $C>1$ and $\left.\delta \in\right] \frac{1}{3}$, $\frac{1}{2}$, then we can apply estimate (23) of Proposition 2.2 and estimate (30) of Proposition 2.5 to get

$$
e_{c}(\vec{u}, \vec{v}, L) \leq \frac{\mathrm{e}^{-\frac{v}{3} \Theta(x)}}{\sqrt{2} \Theta(x)^{\frac{1}{6}}}\left(\frac{\alpha-1}{\alpha+1}\right)^{\frac{1}{4}}\left(1+\frac{2^{\frac{1}{4}} \sqrt{\alpha}}{\sqrt{\alpha-1}} \frac{\Theta(x)^{\frac{1}{6}}}{\sqrt{x}}+\mathcal{O}\left(\frac{1}{v^{1-2 \delta}}\right)\right), \quad x=\frac{v}{L+\frac{1}{2}} .
$$

Let $\epsilon$ be some small positive number,we have

$$
\frac{\mathrm{e}^{-\frac{v}{3} \Theta(x)}}{\sqrt{2} \Theta(x)^{\frac{1}{6}}}\left(\frac{\alpha-1}{\alpha+1}\right)^{\frac{1}{4}}=\epsilon \quad \Longleftrightarrow \quad \Theta(x)=\frac{1}{2 v} W\left(\frac{(\alpha-1)^{\frac{3}{2}} v}{4(\alpha+1)^{\frac{3}{2}} \epsilon^{6}}\right) .
$$

When $v$ is large, $\Theta(x)$ goes to 0 , and $x$ goes to 1 and, following the same lines as what we did previously, we finally get an asymptotic development for the value $L_{\pi}(v, \alpha, \epsilon)$ that gives an error of $\epsilon$

$$
L_{\pi}(v, \alpha, \epsilon)+\frac{1}{2}=v+\left(\frac{1}{2}\right)^{5 / 3} v^{1 / 3} W^{2 / 3}\left(\frac{K(\alpha) v}{4 \epsilon^{6}}\right) \quad \text { with } K(\alpha)=\left(\frac{\alpha-1}{\alpha+1}\right)^{3 / 2} .
$$

\subsection{The case $\hat{\mathbf{u}} \cdot \hat{\mathbf{v}}=1$}

In this case, $P_{\ell}(\hat{u} \cdot \hat{v})=1$ and

$$
e_{c}(\vec{u}, \vec{v}, L)=|\vec{u}+\vec{v}|\left|\sum_{\ell=L+1}^{\infty}(2 \ell+1)(-1)^{\ell} y_{\ell}(u) j_{\ell}(v)\right| .
$$

Using Proposition 2.8, and $\vec{u}+\vec{v}=(\alpha-1) \vec{v}$, we derive the explicit form of the series,

$$
e_{c}(\vec{u}, \vec{v}, L)=\frac{\pi}{2} \sqrt{u v}\left|Y_{L+\frac{3}{2}}(u) J_{L+\frac{1}{2}}(v)+Y_{L+\frac{1}{2}}(u) J_{L+\frac{3}{2}}(v)\right|, \quad \text { when } \hat{u} \cdot \hat{v}=1
$$

and,

$$
\left\{\begin{aligned}
e_{c}(\vec{u}, \vec{v}, L) \leq & \left(\sqrt{\frac{\pi v}{2}} J_{L+\frac{1}{2}}(v)\right)\left(\sqrt{\frac{\pi u}{2}}\left|H_{L+\frac{3}{2}}^{(1)}(u)+H_{L+\frac{1}{2}}^{(1)}(u)\right|\right) \\
& +\left(\sqrt{\frac{\pi v}{2}}\left(J_{L+\frac{3}{2}}(v)-J_{L+\frac{1}{2}}(v)\right)\left(\sqrt{\frac{\pi u}{2}}\left|H_{L+\frac{1}{2}}^{(1)}(u)\right|\right),\right.
\end{aligned}\right.
$$

which is similar to the previous case, except for the difference of signs. The same reasoning as previously can be repeated. The final result for the asymptotic formula is

$$
L_{0}(v, \alpha, \epsilon)+\frac{1}{2}=v+\left(\frac{1}{2}\right)^{5 / 3} v^{1 / 3} W^{2 / 3}\left(\frac{K(\alpha) v}{4 \epsilon^{6}}\right), \quad \text { with } \quad K(\alpha)=\left(\frac{\alpha+1}{\alpha-1}\right)^{3 / 2} .
$$

This is confirmed by numerical results, as shown in Table 3 where we give the values of $L^{1}=L^{\text {actual }}, L^{2}=L^{\text {ours }}$, and the difference $\Delta L=L^{2}-L^{\text {ours }}, L^{2}=L^{\text {ours }}$ being chosen according to 74 , and $L^{1}=L^{\text {actual }}$ being the smallest truncation integer obtained experimentally to achieve

$$
|\alpha \vec{v}+\vec{v}|\left|\sum_{\ell=L+1}^{\infty}(2 \ell+1)(-1)^{\ell} j_{\ell}(v) h_{\ell}^{(1)}(\alpha v)\right| \leq \epsilon .
$$

The comparison is excellent since the two integers are equal almost everywhere. 
TABLE 3. Comparison between the truncation integers $L: L^{2}$ corresponds to the asymptotic formula (74), $L^{1}$ is the actual truncation integer.

\begin{tabular}{|c||c|c|c|c|c|}
\hline & $v=200$ & $v=1000$ & $v=10^{4}$ & $v=10^{5}$ & $v=10^{6}$ \\
\hline \hline$\alpha=\frac{2}{\sqrt{3}}$ & $L^{1}=218$ & $L^{1}=1033$ & $L^{1}=10073$ & $L^{1}=100165$ & $L^{1}=1000370$ \\
$\epsilon=10^{-2}$ & $L^{2}=219$ & $L^{2}=1033$ & $L^{2}=10073$ & $L^{2}=100165$ & $L^{2}=1000370$ \\
& $\Delta L=1$ & $\Delta L=0$ & $\Delta L=0$ & $\Delta L=0$ & $\Delta L=0$ \\
\hline$\alpha=\frac{2}{\sqrt{3}}$ & $L^{1}=234$ & $L^{1}=1056$ & $L^{1}=10122$ & $L^{1}=100268$ & $L^{1}=1000589$ \\
$\epsilon=10^{-5}$ & $L^{2}=232$ & $L^{2}=1055$ & $L^{2}=10122$ & $L^{2}=100268$ & $L^{2}=1000589$ \\
& $\Delta L=2$ & $\Delta L=1$ & $\Delta L=0$ & $\Delta L=0$ & $\Delta L=0$ \\
\hline$\alpha=2$ & $L^{1}=217$ & $L^{1}=1031$ & $L^{1}=10070$ & $L^{1}=100158$ & $L^{1}=1000356$ \\
$\epsilon=10^{-2}$ & $L^{2}=217$ & $L^{2}=1031$ & $L^{2}=10070$ & $L^{2}=100158$ & $L^{2}=1000356$ \\
& $\Delta L=0$ & $\Delta L=0$ & $\Delta L=0$ & $\Delta L=0$ & $\Delta L=0$ \\
\hline$\alpha=2$ & $L^{1}=231$ & $L^{1}=1054$ & $L^{1}=100119$ & $L^{1}=100263$ & $L^{1}=1000578$ \\
$\epsilon=10^{-5}$ & $L^{2}=231$ & $L^{2}=1054$ & $L^{2}=100119$ & $L^{2}=100263$ & $L^{2}=1000578$ \\
& $\Delta L=0$ & $\Delta L=0$ & $\Delta L=0$ & $\Delta L=0$ & $\Delta L=0$ \\
\hline
\end{tabular}

\subsection{The case $|\hat{\mathbf{u}} \cdot \hat{\mathbf{v}}|=|\cos \beta|<1$}

The reasoning that was done for the Jacobi-Anger series in [6] is difficult to repeat here, since we do not have an appropriate formula at our disposal, like Christoffel-Darboux formula, to apply Abel's transformation. However, we can give some elements which show, in an intuitive way, a similarity between both series. First, we note that the sequence $\left(P_{\ell}(\cos \beta)\right)$ is asymptotically equivalent to a trigonometric sequence with a pulsation $\beta$ : the following development can be found for example in ([1], p. 336)

$$
P_{\ell}(\cos \beta)=\sqrt{\frac{2}{\pi \ell \sin \beta}} \cos \left(\left(\ell+\frac{1}{2}\right) \beta-\frac{\pi}{4}\right)+\mathcal{O}\left(\ell^{-1}\right) .
$$

Moreover the sequence $i^{\ell}=\mathrm{e}^{i \ell \frac{\pi}{2}}$ is also trigonometric, with pulsation $\frac{\pi}{2}$. The result concerning Jacobi-Anger consisted in using the fact that, in most of the cases, the sequence $\left((2 \ell+1) i^{\ell} j_{\ell}(v) P_{\ell}(\cos \beta)\right)$ was regularly oscillating while softly diminishing in modulus. Special cases were those when both pulsations were compensating one another, that is to say $\beta \pm \frac{\pi}{2} \equiv 0(\bmod 2 \pi)$.

Here we must consider sequences $(-1)^{\ell}=\mathrm{e}^{i \ell \pi},\left(P_{\ell}(\cos \beta)\right)$ with $\cos \beta=\hat{u} \cdot \hat{v}$ and $\left(Y_{\ell}(u)\right)$. In the Appendix, it is shown that $\left(Y_{\ell}(u)\right)$ is equivalent to a trigonometric sequence with pulsation $\gamma=\arccos \frac{1}{\alpha}$, at least in an area of size $v^{1 / 3}$ around an index. Then we can imagine that the only parameters incurring a non-oscillating behaviour are those satisfying

$$
\pi \pm \beta \pm \gamma \equiv 0 \quad(\bmod 2 \pi) \text { or, equivalently, } \cos \beta=-\frac{1}{\alpha}
$$

Then two different cases should appear:

- If the parameters satisfy (76) then the general term of $e_{c}$ asymptotically keeps a constant sign, and we can deal with absolute values by using the bound (6) as for Jacobi-Anger. Then we obtain an asymptotic behaviour involving a constant argument in the Lambert function.

- Otherwise, the remainder of the series behaves as its first term, and the error goes to 0 if $L=[v]$.

Numerical tests confirm all these conjectures: Figure 7 shows the error variations with respect to $v$ when $L=v+3 v^{1 / 3}$. We take $\alpha=\alpha_{0}=\frac{2}{\sqrt{3}}$ and we consider three different values of $\beta$. When $\cos \beta=1$, the error 


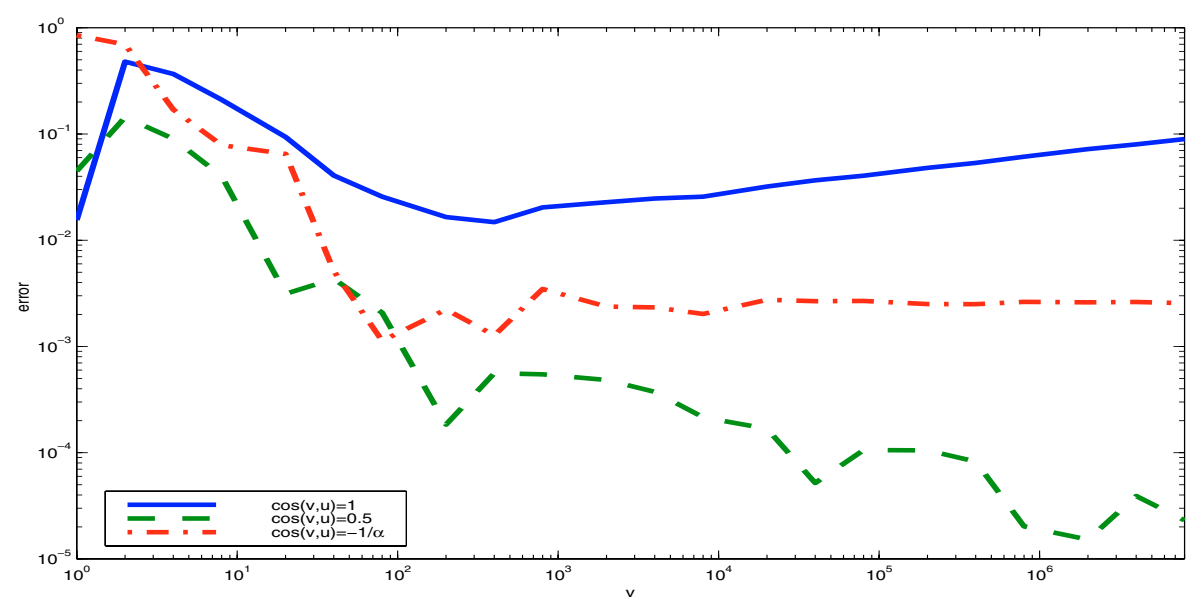

Figure 7. Truncation error for formula $L=v+3 v^{\frac{1}{3}}$ with respect to $v, \hat{v} \cdot \hat{u}$ being equal to 1 , 0.5 and $-\frac{\sqrt{3}}{2}$.

increases softly, as suggested in the previous section. When $\cos \beta=0.5$, the error decreases (this is the most common case). Lastly, when $\cos \beta=-\frac{\sqrt{3}}{2}$, the error converges, exactly as we suggested.

Since the oscillations of $(-1)^{\ell},\left(P_{\ell}(\cos \beta)\right)$ and $\left(Y_{\ell}(u)\right)$ seem to counterbalance when $\cos \beta=-\frac{1}{\alpha}$, then bounding these sequences by their absolute values becomes nearly optimal. By doing it, using (6) leads to

$$
e_{c}(\vec{u}, \vec{v}, L) \leq \sqrt{\frac{2}{\pi v|\sin \beta|}} \frac{|\vec{u}+\vec{v}|}{(\alpha+1) v} e_{c}^{a b s}(u, v, L)
$$

It is easy to prove that $|\vec{u}+\vec{v}|=v \sqrt{\alpha^{2}+1+2 \alpha \cos \beta}=v \sqrt{\alpha^{2}-1}$, therefore

$$
e_{c}(\vec{u}, \vec{v}, L) \leq \sqrt{\frac{2}{\pi v}} \frac{\sqrt{\alpha}\left(\alpha^{2}-1\right)^{1 / 4}}{(\alpha+1)} e_{c}^{a b s}(u, v, L)
$$

By using the results on $e_{c}^{a b s}$, we easily deduce the following asymptotic formula for $L_{\beta=\arccos (-1 / \alpha)}$ :

$$
L_{\beta}(v, \alpha, \epsilon)+\frac{1}{2}=v+\frac{1}{2}\left(\frac{3}{2}\right)^{2 / 3} v^{1 / 3} W^{2 / 3}\left(\frac{4}{3 \pi \epsilon^{2}}\right)
$$

\section{Asymptotic analysis of the truncation integer for the Gegenbauer Series}

In this paragraph, we show that the Legendre Polynomials play a role in the asymptotic value of the truncation integer $L$ for the Gegenbauer series. In a way, bounding the Gegenbauer Series by the series of the absolute values of its terms, then, bounding $\left|P_{\ell}(t)\right|$ by 1 (what is commonly done in the literature) leads to overestimate $L$. Our analysis will rest on the following estimates for Legendre Polynomials, (cf. [16], p. 1047)

$$
\max _{t \geq 0}\left|P_{\ell+1}(t)-P_{\ell}(t)\right| \leq \frac{C_{+}}{\sqrt{\ell+\frac{1}{2}}}, \quad \max _{t \leq 0}\left|P_{\ell+1}(t)+P_{\ell}(t)\right| \leq \frac{C_{-}}{\sqrt{\ell+\frac{1}{2}}}, \quad \max _{|t| \leq 1}\left|P_{\ell+1}(t)+P_{\ell-1}(t)\right| \leq \frac{C_{0}}{\sqrt{\ell+\frac{1}{2}}}
$$

as well as the Bernstein inequality (6). 

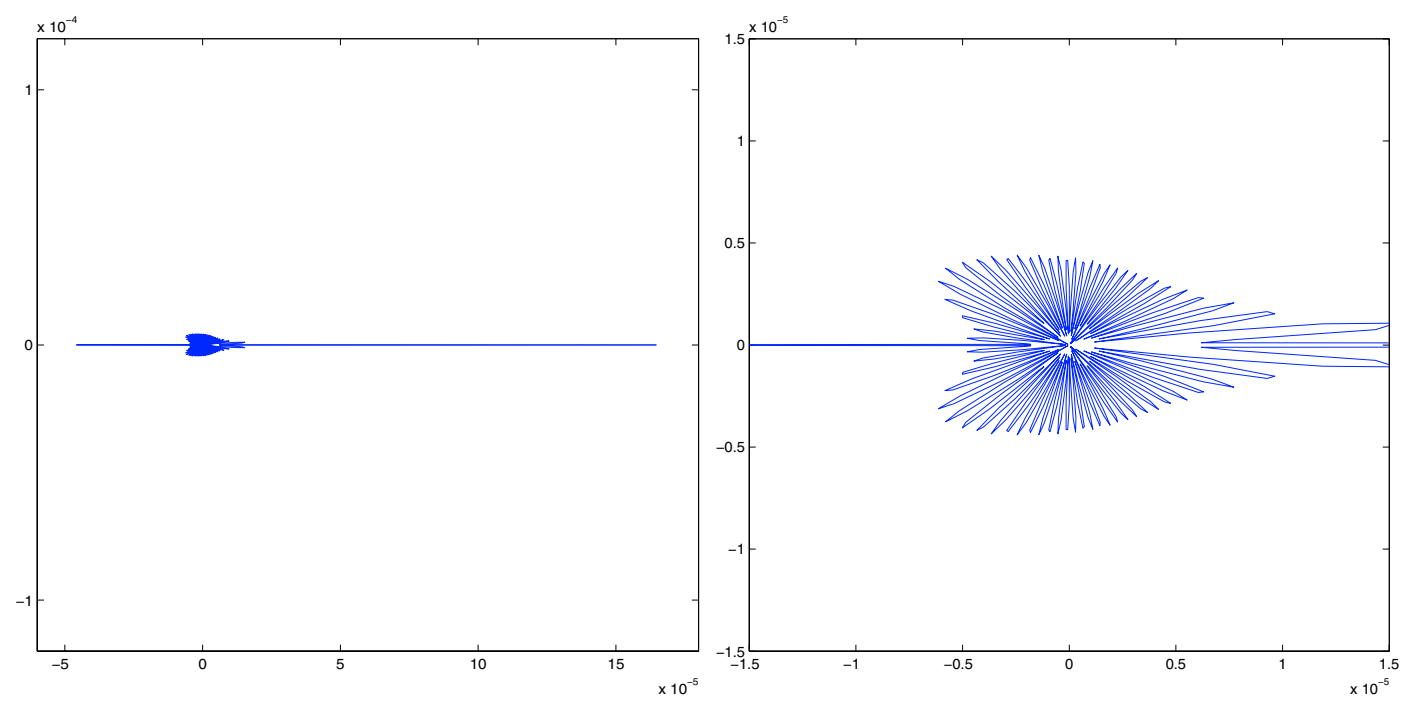

Figure 8. General (left) and close (right) views of curve $\beta \rightarrow \mathrm{e}^{i \beta} e_{c}(\vec{u}, \vec{v}, L)$ with $\vec{v}=v \hat{v}$, $\hat{u} \cdot \hat{v}=\cos \beta, v=1000$ and $L=1046$.

\subsection{Statement of the result}

Theorem 6.1. Let $\alpha_{0}$ be a number greater than 1, $v_{\max }$ a positive number, we consider the set

$$
\Omega=\left\{(\vec{v}, \vec{u}),|\vec{u}| \geq \alpha_{0} v_{\max },|\vec{v}| \leq v_{\max }\right\} .
$$

Let $\epsilon$ be a small positive number, we define $L_{\mathrm{unif}}\left(v_{\max }, \alpha_{0}, \epsilon\right)$ as $\left[\frac{v_{\max }}{x_{\epsilon}\left(v_{\max }\right)}-\frac{1}{2}\right]+1$ where $x_{\epsilon}\left(v_{\max }\right)$ is the unique solution of

$$
\Theta\left(x_{\epsilon}\left(v_{\max }\right)\right)=\frac{1}{2 v} W\left(\left(\frac{\alpha_{0}+1}{\alpha_{0}-1}\right)^{\frac{3}{2}} \frac{v_{\max }}{4 \epsilon^{6}}\right),
$$

where $W(x)$ is Lambert's function (see (17)) and $\Theta(x)$ is the function defined in (31). Then we have

$$
\lim _{v_{\max } \rightarrow \infty} \sup _{u, v \in \Omega} e_{c}\left(\vec{u}, \vec{v}, L_{\text {unif }}\left(v_{\max }, \alpha_{0}, \epsilon\right)\right) \leq \epsilon
$$

Moreover, $L_{\text {unif }}\left(v_{\max }, \alpha_{0}, \epsilon\right)$ satisfies to the following asymptotic expansion

$$
L_{\text {unif }}\left(v_{\max }, \alpha_{0}, \epsilon\right)+\frac{1}{2}=v_{\max }+\left(\frac{1}{2}\right)^{\frac{5}{3}}\left(v_{\max }\right)^{\frac{1}{3}} W^{\frac{2}{3}}\left(\left(\frac{\alpha_{0}+1}{\alpha_{0}-1}\right)^{\frac{3}{2}} \frac{v_{\max }}{4 \epsilon^{6}}\right)+\ldots
$$

This theorem says that, when $v_{\max }$ is large, the maximum of the error over the set $\Omega$ occurs when the two vectors point toward the same direction. Note that this result is not directly applicable to FMM since the set $\Omega$ is not a cube in $v$ (actually it is when the line connecting centers of cubes is a diagonal). An even more complicated analysis should be done to generalize our result to this case.

This result and those of the previous section are illustrated in Figure 8 where, for a given $v=1000$, we plot in polar coordinates the error $e_{c}(\vec{u}, \vec{v}, L)$ versus the angle of aperture $\beta$, with $\alpha_{0}=\frac{2}{\sqrt{3}}, u=\alpha_{0} v$ and $L=1046$. The error reaches its maximum value for $\beta=0$ and $\pi$ and a local maximum can be seen for $\beta= \pm \frac{5 \pi}{6}= \pm \arccos -\frac{1}{\alpha_{0}}$. This numerical result agrees with our analysis. 

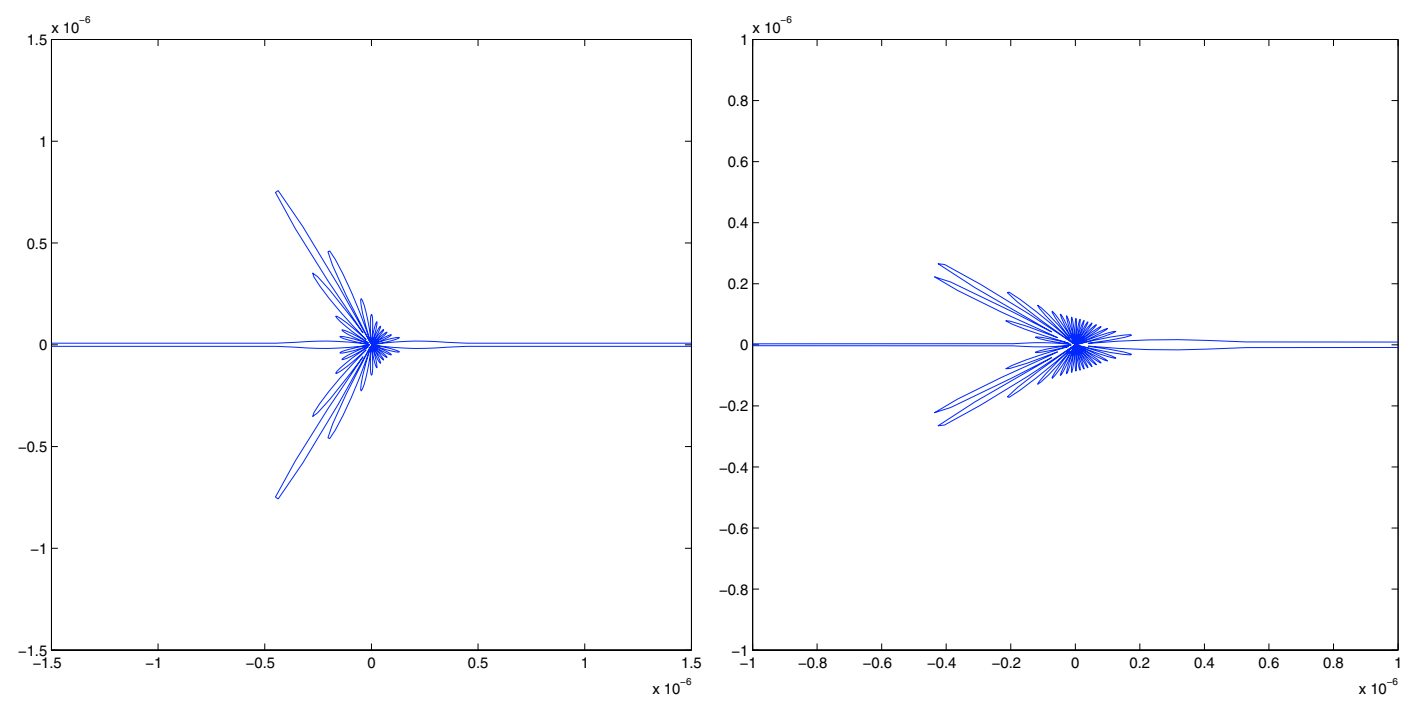

Figure 9. Close views of curves $\beta \rightarrow \mathrm{e}^{i \beta} e_{c}(\vec{u}, \vec{v}, L)$ with $\vec{v}=v \hat{v}, \hat{u} \cdot \hat{v}=\cos \beta, v=70000$ and $L=70220$ for $\alpha_{0}=2$ (left) and $\alpha_{0}=\frac{2}{\sqrt{3}}$ (right).

In Figure 9, the same kind of illustration is given for two different values of $\alpha_{0}$. The left curve is obtained for $\alpha_{0}=2$, whereas $\alpha_{0}=\frac{2}{\sqrt{3}}$ for the right one. In both cases $v=70000$ and $L=70220$. The error reaches its maximum value for $\beta=0$ and $\pi$ and a local maximum can be seen for $\beta= \pm \frac{2 \pi}{3}= \pm \arccos -\frac{1}{2}$ in the left picture and for $\beta= \pm \frac{5 \pi}{6}= \pm \arccos -\frac{\sqrt{3}}{2}$ in the right picture. Notice that the maxima are emphasized with the increase of $v$.

\subsection{Proof of Theorem 6.1}

\subsubsection{Basic ingredients for the proof}

In what follows, $L$ stands for $L_{\text {unif }}\left(v_{\max }, \alpha_{0}, \epsilon\right), \Theta$ stands for $\Theta\left(x_{\epsilon}\left(v_{\max }\right)\right), t$ for $-\hat{u} \cdot \hat{v}$ and $x_{L}$ is the ratio $\frac{v_{\max }}{L+\frac{1}{2}} \leq x_{\epsilon}\left(v_{\max }\right)$. The definition of $x_{\epsilon}\left(v_{\max }\right)$ and the decrease of function $\Theta(x)$ provides:

$$
\frac{\mathrm{e}^{-\frac{v_{\max }}{3} \Theta\left(x_{L}\right)}}{\sqrt{2} \Theta\left(x_{L}\right)^{\frac{1}{6}}}\left(\frac{\alpha_{0}-1}{\alpha_{0}+1}\right)^{\frac{1}{4}} \leq \epsilon
$$

Our proof relies on two different expressions for $e_{c}$ that allow us to treat differently the case $t$ not in the neighborhood of \pm 1 and the case $t$ close to \pm 1 : in one case, we use Bernstein estimate (6) to get

$$
\left|e_{c}(\vec{u}, \vec{v}, L)\right| \leq \frac{C_{b}}{\left(1-t^{2}\right)^{\frac{1}{4}}} \frac{e_{c}^{a b s}(u, v, L)}{\sqrt{v_{\max }}}, \quad C_{b}=\sqrt{\frac{2}{\pi}}
$$

while in the other one we start from the very expression of $e_{c}$

$$
\left|e_{c}(\vec{u}, \vec{v}, L)\right|=\frac{\sqrt{\alpha^{2}-2 \alpha t+1}}{|1-\alpha t|} \frac{\pi}{2 \sqrt{\alpha}}\left|(1-\alpha t) \sum_{\ell=L+1}^{\infty}(2 \ell+1) J_{\ell+\frac{1}{2}}(v) Y_{\ell+\frac{1}{2}}(u) P_{\ell}(t)\right|
$$


then use Proposition 2.7 to get

$$
\left|e_{c}(\vec{u}, \vec{v}, L)\right| \leq \frac{\sqrt{\alpha^{2}-2 \alpha t+1}}{|1-\alpha t|}\left(\frac{\pi}{2 \sqrt{\alpha}}\left|S_{L}(u, v)\right|+\frac{\pi}{2 \sqrt{\alpha}}\left|R_{L}(u, v)\right|\right),
$$

where $S_{L}(u, v)$ and $R_{L}(u, v)$ are defined in 2.7 with the symbols in $\mathcal{B}$. $(u)$ replaced by $Y$. $(u)$. From the bounds $\frac{v}{2}\left(J_{\ell-\frac{1}{2}}(v)-J_{\ell+\frac{3}{2}}(v)+J_{\ell+\frac{1}{2}}(v) / v\right) \leq(\ell+1) J_{\ell+\frac{1}{2}}(v) \leq(2 \ell+1) J_{\ell+\frac{1}{2}}(v),(78)$ for $P_{\ell+1}(t)-P_{\ell-1}(t)$, and $\alpha /(\alpha+1) \leq$ 1 , we easily find that

$$
\frac{\pi}{2 \sqrt{\alpha}}\left|S_{L}(u, v)\right| \leq C_{0} \frac{e_{c}^{a b s}(u, v, L)}{\sqrt{v_{\max }}} .
$$

The first point of the proof is that when $L$ is chosen as we did, $e_{c}^{a b s}(u, v) / \sqrt{v_{\max }}$ goes to zero with $v_{\max }$. The precise result is as follows: if $\delta$ is some number in $] \frac{1}{3}, \frac{1}{2}\left[\right.$ and $v_{\max }$ is large enough, then there exists some pure constant $C$ such that

$$
\sup _{u \geq \alpha_{0} v_{\max }, 0 \leq v \leq v_{\max }} \frac{e_{c}^{a b s}(u, v)}{\sqrt{v_{\max }}} \leq \frac{C}{\xi\left(v_{\max }\right)^{\frac{1}{6}}}\left(1+\mathcal{O}\left(\frac{1}{v_{\max }^{1-2 \delta}}\right)\right) \epsilon, \text { with } \xi(v)=v W^{2}\left(\left(\frac{1+\alpha_{0}}{1-\alpha_{0}}\right)^{\frac{3}{2}} \frac{v}{4 \epsilon^{6}}\right) .
$$

To prove that, we split the series defining $e_{c}^{a b s}(u, v)$ into two parts, one with $\ell$ running from $L+1$ to the integer $L_{a b s}$ defined in Theorem 4.1 and the other with $\ell$ going from $L_{a b s}+1$ to infinity. This can be done because $L_{a b s}$ is asymptotically greater than $L$ (easy to deduce from the properties of Lambert's function). The second series is bounded by $2 \epsilon$ when $v_{\max }$ is large enough, so it can be incorporated into the negligible part of the estimate. For the first series, we bound each term $\left|Y_{\ell+\frac{1}{2}}(u)\right| \leq\left|H_{\ell+\frac{1}{2}}^{(1)}(u)\right|$ via (21) and the remainder series by (32). We get

$$
\frac{\pi}{2} \frac{1+\alpha}{\sqrt{\alpha}}\left(\sum_{\ell=L+1}^{L_{a b s}}(2 \ell+1) J_{\ell+\frac{1}{2}}(v)\left|Y_{\ell+\frac{1}{2}}(u)\right|\right) \leq \frac{1+\alpha_{0}}{\sqrt{\alpha_{0}}\left(\alpha_{0}^{2}-1\right)^{\frac{1}{4}}} \frac{\mathrm{e}^{-\frac{v_{\max }}{3} \Theta\left(x_{L}\right)}}{\sqrt{\Theta\left(x_{L}\right)}}\left(1+\mathcal{O}\left(\frac{1}{v_{\max }^{1-2 \delta}}\right)\right) .
$$

The result follows from

$$
\frac{1+\alpha_{0}}{\sqrt{\alpha_{0}}\left(\alpha_{0}^{2}-1\right)^{\frac{1}{4}}} \frac{\mathrm{e}^{-\frac{v_{\max }}{3} \Theta\left(x_{L}\right)}}{\sqrt{v_{\max } \Theta\left(x_{L}\right)}} \leq \frac{\sqrt{2+\frac{2}{\alpha_{0}}}}{\sqrt{v_{\max }} \Theta^{\frac{1}{3}}}\left(\frac{\mathrm{e}^{-\frac{v_{\max }}{3}} \Theta}{\sqrt{2} \Theta \frac{1}{6}}\left(\frac{1+\alpha_{0}}{1-\alpha_{0}}\right)^{\frac{1}{4}}\right) \leq \frac{C}{\xi\left(v_{\max }\right)^{\frac{1}{6}}} \epsilon .
$$

\subsubsection{Sketch of the proof}

We have now all the elements to write our proof. When $t$ is not in the neighborhood of \pm 1 , we use (82) and (84) to get

$$
\left|e_{c}(\vec{u}, \vec{v}, L)\right| \leq \frac{C_{0} C}{\xi\left(v_{\max }\right)^{\frac{1}{6}}\left(1-t^{2}\right)^{\frac{1}{4}}}(1+\cdots) \epsilon,
$$

and this quantity is less than $(1+\cdots) \epsilon$ when

$$
|1 \pm t| \geq \frac{\left(C_{0} C\right)^{4}}{2 \xi\left(v_{\max }\right)^{\frac{2}{3}}}
$$

Assume now that $t$ lies in the neighborhood of \pm 1 defined in (85); we have

$$
\frac{\sqrt{\alpha^{2}-2 \alpha t+1}}{|1-\alpha t|}=1+\mathcal{O}(|1 \pm t|) \text {, uniformly in } \alpha \geq \alpha_{0} .
$$


Besides, the term $\frac{\pi}{2 \sqrt{\alpha}}\left|S_{L}(u, v)\right|$ is uniformly negligible according to both (83) and (84), and the main contribution is $\frac{\pi}{2 \sqrt{\alpha}}\left|R_{L}(u, v)\right|$, that is

$$
\begin{gathered}
\left|e_{c}(\vec{u}, \vec{v}, L)\right| \lesssim \frac{\pi}{2 \sqrt{\alpha}}\left|R_{L}(u, v)\right|=\frac{\pi}{2} \sqrt{u v}\left|Y_{L+\frac{1}{2}}(u) J_{L+\frac{3}{2}}(v) P_{L+1}(t)-Y_{L+\frac{3}{2}}(u) J_{L+\frac{1}{2}}(v) P_{L}(t)\right| \leq \sum_{i=1}^{4} T_{i} \text {, with } \\
T_{1}=\left|\frac{P_{L+1}(t)+P_{L}(t)}{2}\right| \sqrt{\frac{\pi u}{2}}\left|H_{L+\frac{3}{2}}^{(1)}(u)-H_{L+\frac{1}{2}}^{(1)}(u)\right| \sqrt{\frac{\pi v}{2}}\left|J_{L+\frac{1}{2}}(v)\right| \\
T_{2}=\left|\frac{P_{L+1}(t)-P_{L}(t)}{2}\right| \sqrt{\frac{\pi u}{2}}\left|H_{L+\frac{3}{2}}^{(1)}(u)+H_{L+\frac{1}{2}}^{(1)}(u)\right| \sqrt{\frac{\pi v}{2}}\left|J_{L+\frac{1}{2}}(v)\right| \\
T_{3}=\left|\frac{P_{L+1}(t)+P_{L}(t)}{2}\right| \sqrt{\frac{\pi u}{2}}\left|H_{L+\frac{1}{2}}^{(1)}(u)\right| \sqrt{\frac{\pi v}{2}}\left|J_{L+\frac{3}{2}}(v)-J_{L+\frac{1}{2}}(v)\right| \\
T_{4}=\left|\frac{P_{L+1}(t)-P_{L}(t)}{2}\right| \sqrt{\frac{\pi u}{2}}\left|H_{L+\frac{1}{2}}^{(1)}(u)\right| \sqrt{\frac{\pi v}{2}}\left|J_{L+\frac{3}{2}}(v)-J_{L+\frac{1}{2}}(v)\right| .
\end{gathered}
$$

We use Propositions 2.5 and 2.2 to bound the terms related to Bessel functions, uniformly over $\Omega$. When $t$ is close to -1 (resp. 1), we use (78) and bound the half sum of two consecutive Legendre polynomials by $C^{+} /\left(2 \sqrt{v_{\max }}\right)$ (resp. by 1 ) and bound the half difference by 1 (resp. by $C^{-} /\left(2 \sqrt{v_{\max }}\right)$ ). Finally, the dominant term is $T_{1}$ when $t$ is close to 1 and $T_{2}$ when $t$ is close to -1 ; the conclusion follows from $T_{1} \leq \epsilon$ when $t \simeq 1$ and $T_{2} \leq\left(\frac{1-\alpha_{0}}{1+\alpha_{0}}\right)^{\frac{1}{2}} \epsilon \leq \epsilon$ when $t \simeq-1$.

Remark 6.2. Once again, the result is not only true for the cosine part of the error, but also for the total complex error, since we always bounded $\left|Y_{\ell+\frac{1}{2}}\right|$ by $\left|H_{\ell+\frac{1}{2}}^{(1)}\right|$.

\section{Appendix A. A Uniform APPROXimation for HANKEL FUnCtions}

\section{A.1. Introduction and statement of the result}

We are interested in deriving a simple approximation of the sequence

$$
\left\{H_{\nu_{v}+p}^{(1)}(\alpha v)\right\}_{p=1, \ldots, q}
$$

where

- $\alpha$ is some real number, $\alpha \geq \alpha_{0}>1$;

- $v$ is a positive large number; by this, we mean that for some $C>0$ and some $\delta, 0<\delta<\frac{1}{2}$, we have

$$
v \geq \frac{3}{2} \text { and } \frac{C}{v^{1-\delta}} \leq \frac{\alpha_{0}-1}{2} ;
$$

- $\nu_{v}$ is approximately equal to $v$ in the sense that

$$
\frac{1}{2} \leq v-1 \leq \nu_{v} \leq v
$$

- $q$ is some integer not too large with respect to $v$; more precisely, we assume that

$$
0 \leq q+1 \leq C v^{\delta}
$$

with the same constants $C>0$ and $\delta, 0<\delta<\frac{1}{2}$ as above. 
Note that our hypothesis imply that

$$
\frac{\nu_{v}+p}{\alpha v} \leq \frac{v+q}{\alpha v} \leq \frac{1}{\alpha}+\frac{C}{\alpha v^{1-\delta}} \leq \frac{1}{\alpha_{0}}+\frac{C}{\alpha_{0} v^{1-\delta}} \leq \frac{1}{2}+\frac{1}{2 \alpha_{0}}<1 \text {, for all } p=0, \ldots q,
$$

and, in particular the argument $u=\alpha v$ of the Hankel function is always larger than its index.

Our claim is that when $v$ is large and $q$ not too large, we can use the approximation

$$
H_{\nu_{v}+p}^{(1)}(\alpha v) \simeq H_{\nu_{v}}^{(1)}(\alpha v) \mathrm{e}^{-i p \arccos \left(\frac{1}{\alpha}\right)}, \quad p=0, \ldots q
$$

Intuitively, this results comes from the well known identity

$$
H_{\nu+p+1}^{(1)}(\alpha v)+H_{\nu+p-1}^{(1)}(\alpha v)=\frac{2(\nu+p)}{\alpha v} H_{\nu+p}^{(1)}(\alpha v),
$$

or equivalently

$$
H_{\nu+p+1}^{(1)}(\alpha v)-\frac{2}{\alpha} H_{\nu+p}^{(1)}(\alpha v)+H_{\nu+p-1}^{(1)}(\alpha v)=2\left(\frac{(\nu+p)}{\alpha v}-\frac{1}{\alpha}\right) H_{\nu+p}^{(1)}(\alpha v) .
$$

To provide a rigorous estimate, we define the sequence

$$
x_{p}=H_{\nu_{v}+p}^{(1)}(\alpha v)-\mathrm{e}^{-i p \arccos \left(\frac{1}{\alpha}\right)} H_{\nu_{v}}^{(1)}(\alpha v) .
$$

We have that

with

$$
\left\{\begin{array}{l}
x_{p+1}-\frac{2}{\alpha} x_{p}+x_{p-1}=f_{p} \\
x_{0}=0, \quad x_{1}=H_{\nu_{v}+1}^{(1)}(\alpha v)-\mathrm{e}^{-i \arccos \left(\frac{1}{\alpha}\right)} H_{\nu_{v}}^{(1)}(\alpha v),
\end{array}\right.
$$

$$
f_{p}=2\left(\frac{\left(\nu_{v}+p\right)}{\alpha v}-\frac{1}{\alpha}\right) H_{\nu_{v}+p}^{(1)}(\alpha v)
$$

We will show that both $x_{1}$ and $f_{p}$ are "small" in the sense that when $v, \nu_{v}, q$ satisfy to (86), (87) and (88),

- $\exists C_{\alpha_{0}}^{\bullet}>0$ such that,

$$
\forall u \geq \alpha_{0} v,\left|x_{1}\right|<\frac{C_{\alpha_{0}}^{\bullet}}{u}
$$

(this will be the topic of Prop. A.5),

- $\exists C_{\alpha_{0}}^{\triangle}>0$ such that,

$$
\forall u \geq \alpha_{0} v, \sum_{p=1}^{q}\left|f_{p}\right|<\frac{2 C_{\alpha_{0}}^{\triangle} C^{2}}{\alpha_{0} \sqrt{u} v^{1-2 \delta}}
$$

(this will be a consequence of Prop. A.4 and the bound (88), the hypothesis of A.4 being satisfied due to both inequality (89) and bound (87)),

and this will imply that $x_{p}$ is small since

- if $x_{p}$ satisfies to (90), $\exists C_{\alpha_{0}}^{\star}>0$ such that

$$
\forall u \geq \alpha_{0} v,\left|x_{p}\right|<C_{\alpha_{0}}^{\star}\left(\left|x_{1}\right|+\sum_{r=1}^{p}\left|f_{q}\right|\right)
$$

(this will be the topic of Proposition A.2). 
More precisely, we will obtain that

$$
\exists C_{\alpha_{0}}>0 \text { such that } \forall u \geq \alpha_{0} v,\left|x_{p}\right|<C_{\alpha_{0}}\left(\frac{1}{\sqrt{v}}+\frac{C^{2}}{v^{1-2 \delta}}\right) \frac{1}{\sqrt{u}}
$$

and this is a pertinent result since, thanks to Proposition A.3, we get

$$
\exists C_{\alpha_{0}}>0 \text {, such that } \forall u \geq \alpha_{0} v, \frac{\left|H_{\nu+p}^{(1)}(\alpha v)-\mathrm{e}^{-i p \cos \left(\frac{1}{\alpha}\right)} H_{\nu}^{(1)}(\alpha v)\right|}{\left|H_{v}^{(1)}(\alpha v)\right|}<C_{\alpha_{0}}\left(\frac{1}{\sqrt{v}}+\frac{C^{2}}{v^{1-2 \delta}}\right) .
$$

Finally, our result is the following

Theorem A.1. Let $C, \delta$ be two non negative real numbers $\delta<\frac{1}{2}$. For all $\alpha_{0}$ greater than 1 , there exists some constants $C_{\alpha_{0}}$ and $V=V_{\alpha_{0}, \delta, C}$ such that

$$
\left\{\begin{array}{c}
\forall \alpha>\alpha_{0}, \quad \forall v \geq V, \forall p<C v^{\delta}, \forall \nu, v \leq \nu \leq v+1 \\
\frac{\left|H_{\nu+p}^{(1)}(\alpha v)-\mathrm{e}^{-i p \arccos \left(\frac{1}{\alpha}\right)} H_{\nu}^{(1)}(\alpha v)\right|}{\left|H_{v}^{(1)}(\alpha v)\right|}<C_{\alpha_{0}}\left(\frac{1}{\sqrt{v}}+\frac{C^{2}}{v^{1-2 \delta}}\right)
\end{array}\right.
$$

\section{A.2. Proof of Theorem A.1}

A.2.1. Some estimate for a two steps sequence

Proposition A.2. Let $\alpha_{0}>1$, there exists some constant $C_{\alpha_{0}}^{\star}$ such that all sequence satisfying to

$$
\left\{\begin{array}{l}
x_{p+1}-\frac{2}{\alpha} x_{p}+x_{p-1}=f_{p} \\
x_{0}=u^{(0)}, \quad x_{1}=u^{(1)}
\end{array}\right.
$$

for some data $u^{(0)}, u^{(1)}$ and $f_{p}$ and some parameter $\alpha>\alpha_{0}$ is bounded according to

$$
\left|x_{p}\right| \leq C_{\alpha_{0}}^{\star}\left(\left|u^{(0)}\right|+\left|u^{(1)}\right|+\sum_{q=1}^{p}\left|f_{q}\right|\right) .
$$

Proof. Let $x_{p}$ be such a sequence, we have

$$
\xi^{2}\left(x_{p+1}-2 x_{p}+x_{p-1}\right)+\left(1-\xi^{2}\right)\left(x_{p+1}+2 x_{p}+x_{p-1}\right)=f_{p},
$$

with

We define the energy

$$
\xi^{2}=\frac{1}{2}\left(1+\frac{1}{\alpha}\right), \quad\left(1-\xi^{2}\right)=\frac{1}{2}\left(1-\frac{1}{\alpha}\right)
$$

$$
\mathcal{E}_{p+\frac{1}{2}}=\xi^{2}\left|x_{p+1}-x_{p}\right|^{2}+\left(1-\xi^{2}\right)\left|x_{p+1}+x_{p}\right|^{2}
$$

the first step of the proof is to get the a priori estimate

$$
\mathcal{E}_{p+\frac{1}{2}}=\mathcal{E}_{p-\frac{1}{2}}+\Re\left(\bar{f}_{p}\left(x_{p+1}-x_{p-1}\right)\right) .
$$

This equality is easily obtained by multiplying each term of the conjugate of the equation by $\left(x_{p+1}-x_{p-1}\right)$,

$$
\left(x_{p+1}-x_{p-1}\right)\left(\xi^{2}\left(\bar{x}_{p+1}-2 \bar{x}_{p}+\bar{x}_{p-1}\right)+\left(1-\xi^{2}\right)\left(\bar{x}_{p+1}+2 \bar{x}_{p}+\bar{x}_{p-1}\right)\right)=\bar{f}_{p}\left(x_{p+1}-x_{p-1}\right),
$$


and by using the relationships

$$
\left\{\begin{array}{l}
\Re\left(\left(x_{p+1}-x_{p-1}\right)\left(\bar{x}_{p+1}-2 \bar{x}_{p}+\bar{x}_{p-1}\right)\right)=\left|x_{p+1}-x_{p}\right|^{2}-\left|x_{p}-x_{p-1}\right|^{2} \\
\Re\left(\left(x_{p+1}-x_{p-1}\right)\left(\bar{x}_{p+1}+2 \bar{x}_{p}+\bar{x}_{p-1}\right)\right)=\left|x_{p+1}+x_{p}\right|^{2}-\left|x_{p}+x_{p-1}\right|^{2}
\end{array}\right.
$$

We deduce that

$$
\mathcal{E}_{p+\frac{1}{2}}=\mathcal{E}_{\frac{1}{2}}+\sum_{q=1}^{p} \Re\left(\bar{f}_{q}\left(x_{q+1}-x_{q-1}\right)\right)
$$

so if

$$
G_{0}=\sqrt{\mathcal{E}_{\frac{1}{2}}}, \quad G_{p}=\left(\mathcal{E}_{\frac{1}{2}}+\sum_{q=1}^{p}\left|f_{q}\right|\left(\left|x_{q+1}-x_{q}\right|+\left|x_{q}-x_{q-1}\right|\right)\right)^{\frac{1}{2}}
$$

then

Now, we calculate

$$
\forall p \geq 0, \quad \xi^{2}\left|x_{p+1}-x_{p}\right|^{2} \leq \mathcal{E}_{p+\frac{1}{2}} \leq G_{p}^{2}
$$

$$
\forall p \geq 1, \quad G_{p}^{2}-G_{p-1}^{2}=\left|f_{p}\right|\left(\left|x_{p+1}-x_{p}\right|+\left|x_{p}-x_{p-1}\right|\right) \leq \frac{1}{\xi}\left|f_{p}\right|\left(G_{p}+G_{p-1}\right),
$$

or equivalently

from which we deduce

$$
G_{p} \leq G_{p-1}+\frac{1}{\xi}\left|f_{p}\right|
$$

We infer that

$$
G_{p} \leq \sqrt{\mathcal{E}_{\frac{1}{2}}}+\frac{1}{\xi} \sum_{q=1}^{p}\left|f_{q}\right|
$$

$$
\left|x_{p+1}-x_{p}\right| \leq \frac{1}{\xi} G_{p} \leq \frac{1}{\xi} \sqrt{\mathcal{E}_{\frac{1}{2}}}+\frac{1}{\xi^{2}} \sum_{q=1}^{p}\left|f_{q}\right| .
$$

In a very similar way, from the inequalities

$$
\left(1-\xi^{2}\right)\left|x_{p+1}+x_{p}\right|^{2} \leq \mathcal{E}_{p+\frac{1}{2}} \leq H_{p}^{2}
$$

with

we deduce that

$$
H_{p}^{2}=\mathcal{E}_{\frac{1}{2}}+\sum_{q=1}^{p}\left|f_{q}\right|\left(\left|x_{q+1}+x_{q}\right|+\left|x_{q}+x_{q-1}\right|\right)
$$

$$
\left|x_{p+1}+x_{p}\right| \leq \frac{1}{\sqrt{1-\xi^{2}}} H_{p} \leq \frac{1}{\sqrt{1-\xi^{2}}} \sqrt{\mathcal{E}_{\frac{1}{2}}}+\frac{1}{\left(1-\xi^{2}\right)} \sum_{q=1}^{p}\left|f_{q}\right| .
$$

From the precise definition of $\mathcal{E}_{\frac{1}{2}}$ we get

$$
\mathcal{E}_{\frac{1}{2}} \leq \max \left(\xi^{2},\left(1-\xi^{2}\right)\right)\left(\left|x_{1}\right|+\left|x_{0}\right|\right)^{2}=\xi^{2}\left(\left|x_{1}\right|+\left|x_{0}\right|\right)^{2},
$$

and the remainder is easy, gathering (95) (96) and (97), we have

$$
2\left|x_{p}\right| \leq\left(\frac{\xi}{\sqrt{1-\xi^{2}}}+1\right)\left(\left|x_{1}\right|+\left|x_{0}\right|\right)+\left(\frac{1}{1-\xi^{2}}+\frac{1}{\xi^{2}}\right) \sum_{q=1}^{p}\left|f_{q}\right|,
$$


and

$$
\left|x_{p}\right| \leq C_{\alpha}^{\star}\left(\left|u^{(0)}\right|+\left|u^{(1)}\right|+\sum_{q=1}^{p}\left|f_{q}\right|\right), \text { with } C_{\alpha}^{\star}=\frac{1}{2} \max \left(1+\sqrt{\frac{\xi^{2}}{1-\xi^{2}}}, \frac{1}{1-\xi^{2}}+\frac{1}{\xi^{2}}\right) .
$$

This ends the proof since

$$
C_{\alpha}^{\star}=\frac{2 \alpha^{2}}{\alpha^{2}-1} \leq C_{\alpha_{0}}^{\star}=\frac{2 \alpha_{0}^{2}}{\alpha_{0}^{2}-1} \quad \text { when } \alpha>\alpha_{0} .
$$

A.2.2. An estimate for a sum of Hankel functions

Proposition A.3. Let $\alpha_{0}>1$, there exists some constant $C_{\alpha_{0}}$ such that all non negative real $\mu$ and $u$ satisfying to

we have

$$
\frac{\mu}{u} \leq \frac{1}{2}+\frac{1}{2 \alpha_{0}}, \quad \mu \geq \frac{1}{2},
$$

$$
\sqrt{\frac{2}{\pi u}} \leq\left|H_{\mu}^{(1)}(u)\right| \leq C_{\alpha_{0}} \frac{1}{\sqrt{u}} .
$$

Proof. According to Watson ([22], Sect. 13.73, p. 447, formula (1))

$$
\frac{2}{\pi u}<J_{\mu}^{2}(u)+Y_{\mu}^{2}(u)=\left|H_{\mu}^{(1)}(u)\right|^{2} \leq \frac{2}{\pi \sqrt{u^{2}-\mu^{2}}},
$$

and $1-\left(\frac{\mu}{u}\right)^{2}$ is smaller than $1-\frac{1}{4}\left(1+\frac{1}{\alpha_{0}}\right)^{2}$.

Proposition A.4. Let $\alpha_{0}>1$, there exists some constant $C_{\alpha_{0}}$ such that for all positive integer $q$ and for all non negative $\nu$, u satisfying to

$$
\frac{\nu+q}{u} \leq \frac{1}{2}+\frac{1}{2 \alpha_{0}}, \quad \nu \geq \frac{1}{2},
$$

then

$$
2 \sum_{p=0}^{q}\left(\frac{\nu+p}{u}-\frac{\nu}{u}\right)\left|H_{\nu+p}^{(1)}(u)\right| \leq C_{\alpha_{0}} \frac{q(q+1)}{u \sqrt{u}} .
$$

If moreover

for some $\alpha \geq \alpha_{0}$ then

$$
\frac{1}{\alpha}-\frac{1}{u} \leq \frac{\nu}{u} \leq \frac{1}{\alpha}
$$

$$
2 \sum_{p=1}^{q}\left(\frac{\nu+p}{u}-\frac{1}{\alpha}\right)\left|H_{\nu+p}^{(1)}(u)\right| \leq C_{\alpha_{0}} \frac{q(q+1)}{u \sqrt{u}} .
$$

Proof. A direct consequence of the previous proposition.

A.2.3. An approximate relationship for two consecutive Hankel functions

Proposition A.5. Let $\alpha_{0}>1$, there exists some constants $C_{\alpha_{0}}^{\diamond}$ and $C_{\alpha_{0}}^{\bullet}$ such that for all $\nu$ and $u$ positive satisfying to

then

$$
\frac{\nu}{u} \leq \frac{1}{2}+\frac{1}{2 \alpha_{0}}, \quad \nu \geq \frac{1}{2}
$$

and, if

$$
\left|\tilde{x}_{1}\right|=\left|H_{\nu+1}^{(1)}(u)-\mathrm{e}^{-i \arccos \frac{\nu}{u}} H_{\nu}^{(1)}(u)\right| \leq \frac{C_{\alpha_{0}}^{\diamond}}{u}
$$

$$
\frac{1}{\alpha}-\frac{1}{u} \leq \frac{\nu}{u} \leq \frac{1}{\alpha}
$$




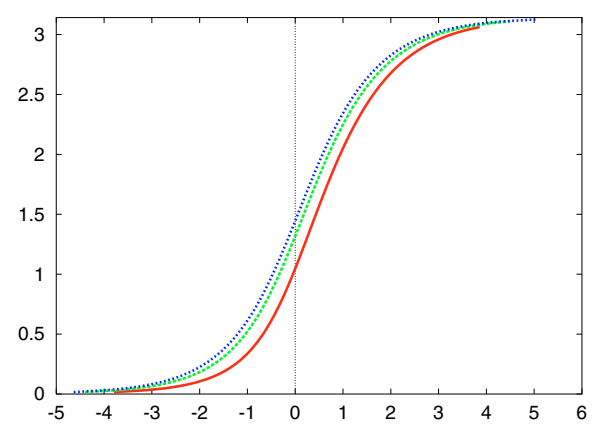

Figure 10. Paths $\Gamma_{\gamma}$ for different values of $\gamma=\arccos \left(\frac{1}{\alpha}\right), \alpha=2,4,8$.

for some $\alpha \geq \alpha_{0}$ then

$$
\left|x_{1}\right|=\left|H_{\nu+1}^{(1)}(u)-\mathrm{e}^{-i \arccos \frac{1}{\alpha}} H_{\nu}^{(1)}(u)\right| \leq \frac{C_{\alpha_{0}}^{\bullet}}{u} .
$$

Proof. We use formula (2) in ([22], Sect. 6.21, p. 178)

$$
H_{\nu}^{(1)}(u)=\frac{1}{i \pi} \int_{-\infty}^{i \pi+\infty} \mathrm{e}^{u \sinh w-\nu w} \mathrm{~d} w
$$

and so

$$
\tilde{x}_{1}=\frac{1}{i \pi} \int_{-\infty}^{i \pi+\infty} \mathrm{e}^{u \sinh w-\nu w}\left(\mathrm{e}^{-w}-\mathrm{e}^{-i \gamma}\right) \mathrm{d} w
$$

where $\gamma$ is the arc cosine of the ratio $\left.\frac{\nu}{u}, \gamma \in\right] 0, \frac{\pi}{2}[$. Following Watson procedure, we choose as path a curve for which

$$
\Im(\sinh w-w \cos \gamma)=\Im(\sinh i \gamma-i \gamma \cos \gamma)
$$

this corresponds to the curve, displayed in Figure 17 ([22], Sect. 8.3, p. 239),

$$
\Gamma_{\gamma}=\left\{w=w_{r}+i v, \cosh w_{r}=\frac{\sin \gamma+(v-\gamma) \cos \gamma}{\sin v}, v \in\right] 0, \pi\left[, w_{r}(v-\gamma)>0\right\}
$$

A parameterization of this curve is

$$
\tau=i(\sin \gamma-\gamma \cos \gamma)-(\sinh w-w \cos \gamma), \quad \tau \geq 0
$$

It is easy to verify that $\tau$ is real and positive on the path: as $w$ travels along the path from $-\infty$ to $\infty+i \pi$, $\tau$ decreases from $+\infty$ to 0 (when $w=i \gamma$ ) and then increases to $+\infty$. We will call $w_{1}(\tau)$ and $w_{2}(\tau)$ the two values of $w$ on the path corresponding to a given value of $\tau$ and it will be supposed that $\Re w_{1}>0$ and $\Re w_{2}<0$. We have

$$
\tilde{x}_{1}=\frac{\mathrm{e}^{i \nu(\tan \gamma-\gamma)}}{i \pi} \int_{0}^{\infty} \mathrm{e}^{-u \tau}\left\{\frac{\mathrm{d} w_{1}}{\mathrm{~d} \tau}\left(\mathrm{e}^{-w_{1}}-\mathrm{e}^{-i \gamma}\right)-\frac{\mathrm{d} w_{2}}{\mathrm{~d} \tau}\left(\mathrm{e}^{-w_{2}}-\mathrm{e}^{-i \gamma}\right)\right\} \mathrm{d} \tau,
$$

with

We simply use the bound

$$
\frac{\mathrm{d} w_{p}}{\mathrm{~d} \tau}=\frac{-1}{\cosh w_{p}-\cos \gamma}
$$

$$
\left|\tilde{x}_{1}\right| \leq \frac{2}{\pi}\left(\int_{0}^{\infty} \mathrm{e}^{-u \tau} \mathrm{d} \tau\right) \sup _{\Gamma_{\gamma}}(|H(w)|), \text { where } H(w)=\frac{\mathrm{e}^{-w}-\mathrm{e}^{-i \gamma}}{\cosh w-\cos \gamma}=\frac{2}{1-\mathrm{e}^{-w-i \gamma}}
$$


We remark that the path $\Gamma_{\gamma}$ lies in the domain $\Omega=\{w, 0<\Im w<\pi\}$. Thanks to the maximum principle, the analytical function $H(w)$ reaches its maximum over $\Omega$ on the boundary of $\Omega$, let for some $w=\lambda$ or $w=i \pi+\lambda$ with $\lambda$ real. A simple calculation shows that it is reached when $\mathrm{e}^{-\lambda}= \pm \cos \gamma$. We get

$$
\sup _{\Gamma_{\gamma}}(|H(w)|) \leq \sup _{0 \leq \Im w \leq \pi} \frac{2}{\mid 1-\mathrm{e}^{-w-i \gamma \mid}}=\frac{2}{|\sin \gamma|}
$$

whence

$$
\left|\tilde{x}_{1}\right| \leq \frac{4}{\pi u|\sin \gamma|}
$$

The estimate (101) is now easily obtained: when $\cos \gamma$ increases from 0 to $\frac{1}{2}+\frac{1}{2 \alpha_{0}}$ the inverse of $|\sin \gamma|$ increases and

$$
\left|\tilde{x}_{1}\right| \leq \frac{C_{\alpha_{0}}^{\diamond}}{u} \text { with } C_{\alpha_{0}}^{\diamond}=\frac{4}{\pi \sqrt{1-\frac{1}{4}\left(1+\frac{1}{\alpha_{0}}\right)^{2}}}
$$

Now, to obtain (103), we set

$$
\delta \phi=\arccos \left(\frac{1}{\alpha}\right)-\arccos \left(\frac{\nu}{u}\right)
$$

Using (102), we get

$$
\delta \phi=\int_{\frac{1}{\alpha}-\frac{1}{u}}^{\frac{1}{\alpha}} \frac{\mathrm{d} t}{\sqrt{1-t^{2}}} \leq \frac{1}{u} \frac{1}{\sqrt{1-\left(\frac{1}{\alpha}\right)^{2}}} \leq \frac{1}{u} \frac{1}{\sqrt{1-\left(\frac{1}{\alpha_{0}}\right)^{2}}}=\frac{C_{\alpha_{0}}}{u} .
$$

It follows that

$$
\left|x_{1}\right| \leq\left|\tilde{x}_{1}\right|+\left|1-\mathrm{e}^{-i \Delta \phi}\right|\left|H_{\nu}^{(1)}(u)\right| \leq \frac{C_{\alpha_{0}}}{u}+\Delta \phi\left|H_{\nu}^{(1)}(u)\right|
$$

and the results follows thanks to (98) and $\nu \geq \frac{1}{2}$ :

$$
\left|x_{1}\right| \leq \frac{C_{\alpha_{0}}^{\diamond}}{u}+\frac{C_{\alpha_{0}}^{\Delta} C_{\alpha_{0}}}{u \sqrt{u}} \leq \frac{C_{\alpha_{0}}^{\bullet}}{u} \quad \text { since } \quad \frac{1}{u} \leq \frac{2 \nu}{u} \leq \alpha_{0}+1
$$

\section{REFERENCES}

[1] M. Abramowitz and I. Stegun, Handbook of Mathematical Functions. Dover, New-York (1964).

[2] S. Amini and A. Profit, Analysis of the truncation errors in the fast multipole method for scattering problems. J. Comput. Appl. Math. 115 (2000) 23-33.

[3] J.A. Barcelo, A. Ruiz and L. Vega, Weighted estimates for the Helmholtz equation and some applications. J. Funct. Anal. 150 (1997) 356-382.

[4] H. Bateman, Higher transcendental Functions. McGraw-Hill (1953).

[5] Q. Carayol, Développement et analyse d'une méthode multipôle multiniveau pour l'électromagnétisme. Ph.D. thesis, Université Paris VI Pierre et Marie Curie, rue Jussieu 75005 Paris (2002).

[6] Q. Carayol and F. Collino, Error estimates in the fast multipole method for scattering problems. part 1: Truncation of the jacobi-anger series. ESAIM: M2AN 38 (2004) 371-394.

[7] T.M. Cherry, Uniform asymptotic formulae for functions with transition points. Trans. AMS 68 (1950) 224-257.

[8] W.C. Chew, J.M. Jin, E. Michielssen and J.M. Song, Fast and Efficient Algorithms in Computational Electromagnetics. Artech House (2001).

[9] R. Coifman, V. Rokhlin and S. Greengard, The Fast Multipole Method for the wave equation: A pedestrian prescription. IEEE Antennas and Propagation Magazine 35 (1993) 7-12.

[10] D. Colton and R. Kress, Inverse Acoustic and Electromagnetic Scattering Theory. Springer-Verlag 93 (1992).

[11] E. Darve, The fast multipole method. I. Error analysis and asymptotic complexity. SIAM J. Numer. Anal. 38 (2000) 98-128 (electronic).

[12] E. Darve, The fast multipole method: Numerical implementation. J. Comput. Physics 160 (2000) 196-240.

[13] E. Darve and P. Havé, Efficient fast multipole method for low frequency scattering. J. Comput. Physics 197 (2004) 341-363. 
[14] B. Dembart and E. Yip, Accuracy of fast multipole methods for maxwell's equations. IEEE Comput. Sci. Engrg. 5 (1998) 48-56.

[15] M.A. Epton and B. Dembart, Multipole translation theory for the three-dimensional Laplace and Helmholtz equations. SIAM J. Sci. Comput. 16 (1995) 865-897.

[16] I.S. Gradshteyn, I.M. Ryzhik, Table of integrals, series, and products, 5th edition. Academic Press (1994).

[17] S. Koc, J. Song and W.C. Chew, Error analysis for the numerical evaluation of the diagonal forms of the scalar spherical addition theorem. SIAM J. Numer. Anal. 36 (1999) 906-921 (electronic).

[18] L. Lorch, Alternative proof of a sharpened form of Bernstein's inequality for Legendre polynomials. Applicable Anal. 14 $(1982 / 83)$ 237-240.

[19] L. Lorch, Corrigendum: "Alternative proof of a sharpened form of Bernstein's inequality for Legendre polynomials" [Appl. Anal. 14 (1982/83) 237-240; MR 84k:26017]. Appl. Anal. 50 (1993) 47.

[20] J.C. Nédélec, Acoustic and Electromagnetic Equation. Integral Representation for Harmonic Problems. Springer-Verlag 144 (2001).

[21] J. Rahola, Diagonal forms of the translation operators in the fast multipole algorithm for scattering problems. BIT 36 (1996) 333-358.

[22] G.N. Watson, A treatise on the theory of Bessel functions. Cambridge University Press (1966).

To access this journal online:

www.edpsciences.org 